\title{
First proton-proton collisions at the LHC as observed with the ALICE detector: measurement of the charged-particle pseudorapidity density at $\sqrt{s}=900 \mathrm{GeV}$
}

The ALICE Collaboration

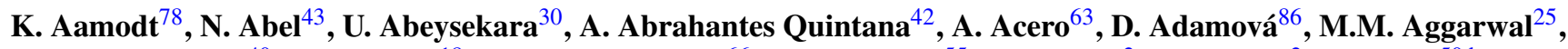
G. Aglieri Rinella ${ }^{40}$, A.G. Agocs ${ }^{18}$, S. Aguilar Salazar ${ }^{66}$, Z. Ahammed Ah $^{55}$ A. Ahmad A N. Ahmad $^{2}$, S.U. Ahn $^{50, b}$, R. Akimoto ${ }^{100}$, A. Akindinov ${ }^{68}$, D. Aleksandrov ${ }^{70}$, B. Alessandro ${ }^{102}$, R. Alfaro Molina ${ }^{66}$, A. Alici ${ }^{13}$, E. Almaráz Aviña ${ }^{66}$, J. Alme ${ }^{8}$, T. Alt ${ }^{43, c}$, V. Altini ${ }^{5}$, S. Altinpinar ${ }^{32}$, C. Andrei ${ }^{17}$, A. Andronic An $^{32}$, G. Anelli ${ }^{40}$, V. Angelov ${ }^{43, c}$, C. Anson ${ }^{27}$, T. Antičić ${ }^{113}$, F. Antinori ${ }^{40, d}$, S. Antinori ${ }^{13}$, K. Antipin ${ }^{37}$, D. Antończyk ${ }^{37}$, P. Antonioli ${ }^{14}$, A. Anzo ${ }^{66}$, L. Aphecetche ${ }^{73}$, H. Appelshäuser ${ }^{37}$, S. Arcelli ${ }^{13}$, R. Arceo ${ }^{66}$, A. Arend ${ }^{37}$, N. Armesto ${ }^{92}$, R. Arnaldi ${ }^{102}$, T. Aronsson ${ }^{74}$, I.C. Arsene ${ }^{78, e}$, A. Asryan ${ }^{98}$, A. Augustinus ${ }^{40}$, R. Averbeck $^{32}$, T.C. Awes ${ }^{76}$, J. Äystö ${ }^{49}$, M.D. Azmi ${ }^{2}$,

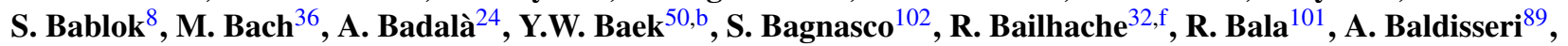
A. Baldit ${ }^{26}$, J. Bán ${ }^{58}$, R. Barbera ${ }^{23}$, G.G. Barnaföldi ${ }^{18}$, L. Barnby ${ }^{12}$, V. Barret ${ }^{26}$, J. Bartke B. F. Barile $^{5}$, M. Basile ${ }^{13}$, V. Basmanov ${ }^{94}$, N. Bastid ${ }^{26}$, B. Bathen ${ }^{72}$, G. Batigne ${ }^{73}$, B. Batyunya ${ }^{35}$, C. Baumann ${ }^{72, f}$, I.G. Bearden ${ }^{28}$,

B. Becker $^{20, \mathrm{~g}}$, I. Belikov ${ }^{99}$, R. Bellwied ${ }^{34}$, E. Belmont-Moreno ${ }^{66}$, A. Belogianni ${ }^{4}$, L. Benhabib ${ }^{73}$, S. Beole $^{101}$, I. Berceanu ${ }^{17}$, A. Bercuci ${ }^{32, h}$, E. Berdermann ${ }^{32}$, Y. Berdnikov ${ }^{39}$, L. Betev ${ }^{40}$, A. Bhasin ${ }^{48}$, A.K. Bhati ${ }^{25}$, L. Bianchi $^{101}$, N. Bianchi ${ }^{38}$, C. Bianchin ${ }^{79}$, J. Bielčík ${ }^{81}$, J. Bielčíková ${ }^{86}$, A. Bilandzic ${ }^{3}$, L. Bimbot ${ }^{77}$, E. Biolcati ${ }^{101}$, A. Blanc ${ }^{26}$, F. Blanco ${ }^{23, i}$, F. Blanco ${ }^{63}$, D. Blau ${ }^{70}$, C. Blume ${ }^{37}$, M. Boccioli ${ }^{40}$, N. Bock ${ }^{27}$, A. Bøgdanov ${ }^{69}$, H. Bøggild $^{28}$, M. Bogolyubsky ${ }^{83}$, J. Bohm ${ }^{96}$, L. Boldizsár ${ }^{18}$, M. Bombara ${ }^{12, j}$, C. Bombonati ${ }^{79, k}$, M. Bondila ${ }^{49}$, H. Borel $^{89}$, V. Borshchov ${ }^{51}$, C. Bortolin ${ }^{79}$, S. Bose ${ }^{54}$, L. Bosisio ${ }^{103}$, F. Bossú ${ }^{101}$, M. Botje ${ }^{3}$, S. Böttger ${ }^{43}$, G. Bourdaud $^{73}$, B. Boyer $^{77}$, M. Braun ${ }^{98}$, P. Braun-Munzinger ${ }^{32,33, c}$, L. Bravina ${ }^{78}$, M. Bregant ${ }^{103,1}$, T. Breitner ${ }^{43}$, G. Bruckner $^{40}$, R. Brun $^{40}$, E. Bruna ${ }^{74}$, G.E. Bruno ${ }^{5}$, D. Budnikov ${ }^{94}$, H. Buesching ${ }^{37}$, K. Bugaev ${ }^{52}$, P. Buncic ${ }^{40}$, O. Busch ${ }^{44}$, Z. Buthelezi ${ }^{22}$, D. Caffarri ${ }^{79}$, X. Cai ${ }^{111}$, H. Caines ${ }^{74}$, E. Camacho ${ }^{64}$, P. Camerini ${ }^{103}$, M. Campbell ${ }^{40}$, V. Canoa $\operatorname{Roman}^{40}$, G.P. Capitani ${ }^{38}$, G. Cara Romeo ${ }^{14}$, F. Carena ${ }^{40}$, W. Carena ${ }^{40}$, F. Carminati ${ }^{40}$,

A. Casanova Díaz ${ }^{38}$, M. Caselle ${ }^{40}$, J. Castillo Castellanos ${ }^{89}$, J.F. Castillo Hernandez ${ }^{32}$, V. Catanescu ${ }^{17}$, E. Cattaruzza ${ }^{103}$, C. Cavicchioli ${ }^{40}$, P. Cerello ${ }^{102}$, V. Chambert ${ }^{77}$, B. Chang $^{96}$, S. Chapeland ${ }^{40}$, A. Charpy ${ }^{77}$, J.L. Charvet ${ }^{89}$, S. Chattopadhyay ${ }^{54}$, S. Chattopadhyay ${ }^{55}$, M. Cherney $^{30}$, C. Cheshkov ${ }^{40}$, B. Cheynis ${ }^{62}$, E. Chiavassa $^{101}$, V. Chibante Barroso ${ }^{40}$, D.D. Chinellato ${ }^{21}$, P. Chochula ${ }^{40}$, K. Choi ${ }^{85}$, M. Chojnacki ${ }^{106}$, P. Christakoglou ${ }^{106}$, C.H. Christensen ${ }^{28}$, P. Christiansen ${ }^{61}$, T. Chujo ${ }^{105}$, F. Chuman ${ }^{45}$, C. Cicalo $^{20}$, L. Cifarelli $^{13}$, F. Cindolo ${ }^{14}$, J. Cleymans ${ }^{22}$, O. Cobanoglu ${ }^{101}$, J.-P. Coffin ${ }^{99}$, S. Coli ${ }^{102}$, A. Colla ${ }^{40}$, G. Conesa Balbastre ${ }^{38}$, Z. Conesa del Valle ${ }^{73, m}$, E.S. Conner ${ }^{110}$, P. Constantin ${ }^{44}$, G. Contin ${ }^{103, \mathrm{k}}$, J.G. Contreras ${ }^{64}$, Y. Corrales Morales ${ }^{101}$, T.M. Cormier ${ }^{34}$, P. Cortese $^{1}$, I. Cortés Maldonado ${ }^{84}$, M.R. Cosentino ${ }^{21}$, F. Costa ${ }^{40}$, M.E. Cotallo ${ }^{63}$, E. Crescio ${ }^{64}$, P. Crochet ${ }^{26}$, E. Cuautle ${ }^{65}$, L. Cunqueiro ${ }^{38}$, J. Cussonneau ${ }^{73}$, A. Dainese ${ }^{59, \text { d }}$, H.H. Dalsgaard ${ }^{28}$, A. Danu ${ }^{16}$, I. Das ${ }^{54}$,

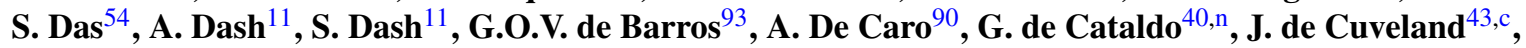
A. De Falco ${ }^{19}$, M. de Gaspari ${ }^{44}$, J. de Groot ${ }^{40}$, D. De Gruttola ${ }^{90}$, A.P. de Haas ${ }^{106}$, N. De Marco ${ }^{102}$, R. de Rooij ${ }^{106}$, S. De Pasquale ${ }^{90}$, G. de Vaux ${ }^{22}$, H. Delagrange ${ }^{73}$, G. Dellacasa ${ }^{1}$, A. Deloff ${ }^{107}$, V. Demanov ${ }^{94}$, E. Dénes $^{18}$, A. Deppman ${ }^{93}$, G. D’Erasmo ${ }^{5}$, D. Derkach ${ }^{98}$, A. Devaux ${ }^{26}$, D. Di Bari ${ }^{5}$, C. Di Giglio ${ }^{5, k}$, S. Di Liberto ${ }^{88}$, A. Di Mauro ${ }^{40}$, P. Di Nezza ${ }^{38}$, M. Dialinas ${ }^{73}$, L. Díaz ${ }^{65}$, R. Díaz ${ }^{49}$, T. Dietel ${ }^{72}$, H. Ding ${ }^{111}$, R. Diviàa ${ }^{40}$, Ø. Djuvsland ${ }^{8}$, G. do Amaral Valdiviesso ${ }^{21}$, V. Dobretsov ${ }^{70}$, A. Dobrin ${ }^{61}$, T. Dobrowolski ${ }^{107}$, B. Dönigus ${ }^{32}$, I. Domínguez $^{65}$, D.M.M. Don ${ }^{46}$, O. Dordic ${ }^{78}$, A.K. Dubey ${ }^{55}$, J. Dubuisson ${ }^{40}$, L. Ducroux ${ }^{62}$, P. Dupieux ${ }^{26}$, A.K. Dutta Majumdar ${ }^{54}$, M.R. Dutta Majumdar ${ }^{55}$, D. Elia ${ }^{6}$, D. Emschermann ${ }^{44, o}$, A. Enokizono ${ }^{76}$, B. Espagnon ${ }^{77}$, M. Estienne ${ }^{73}$, D. Evans ${ }^{12}$,

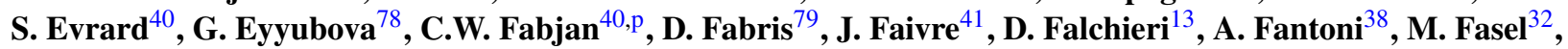
R. Fearick ${ }^{22}$, A. Fedunov ${ }^{35}$, D. Fehlker ${ }^{8}$, V. Fekete ${ }^{15}$, D. Felea ${ }^{16}$, B. Fenton-Olsen ${ }^{28, q}$, G. Feofilov ${ }^{98}$,

A. Fernández Téllez $^{84}$, E.G. Ferreiro ${ }^{92}$, A. Ferretti ${ }^{101}$, R. Ferretti ${ }^{1, r}$, M.A.S. Figueredo ${ }^{93}$, S. Filchagin ${ }^{94}$, R. Fini ${ }^{6}$,

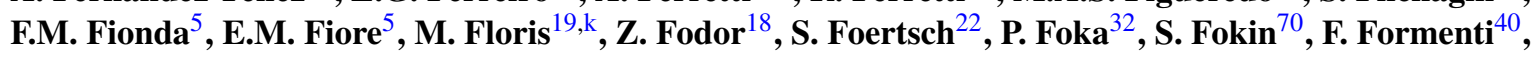




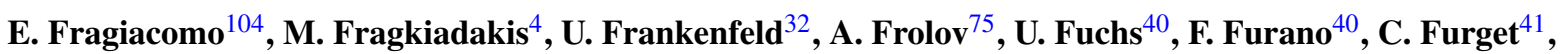
M. Fusco Girard ${ }^{90}$, J.J. Gaardhøje ${ }^{28}$, S. Gadrat ${ }^{41}$, M. Gagliardi ${ }^{101}$, A. Gago ${ }^{64, s}$, M. Gallio ${ }^{101}$, P. Ganoti ${ }^{4}$, M.S. Ganti ${ }^{55}$, C. Garabatos ${ }^{32}$, C. García Trapaga ${ }^{101}$, J. Gebelein ${ }^{43}$, R. Gemme ${ }^{1}$, M. Germain ${ }^{73}$, A. Gheata ${ }^{40}$, M. Gheata ${ }^{40}$, B. Ghidini ${ }^{5}$, P. Ghosh ${ }^{55}$, G. Giraudo ${ }^{102}$, P. Giubellino $^{102}$, E. Gladysz-Dziadus ${ }^{29}$, R. Glasow ${ }^{72, t}$, P. Glässel ${ }^{44}$, A. Glenn ${ }^{60}$, R. Gomez ${ }^{31}$, H. González Santos ${ }^{84}$, L.H. González-Trueba ${ }^{66}$, P. González-Zamora ${ }^{63}$, S. Gorbunov ${ }^{43, c}$, Y. Gorbunov ${ }^{30}$, S. Gotovac ${ }^{97}$, H. Gottschlag ${ }^{72}$, V. Grabski ${ }^{66}$, R. Grajcarek ${ }^{44}$, A. Grelli ${ }^{106}$,

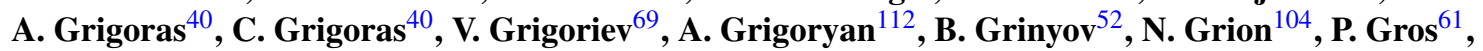
J.F. Grosse-Oetringhaus ${ }^{40}$, J.-Y. Grossiord ${ }^{62}$, R. Grosso $^{80}$, C. Guarnaccia $^{90}$, F. Guber ${ }^{67}$, R. Guernane ${ }^{41}$, B. Guerzoni ${ }^{13}$, K. Gulbrandsen ${ }^{28}$, H. Gulkanyan ${ }^{12}$, T. Gunji ${ }^{100}$, A. Gupta ${ }^{48}$, R. Gupta ${ }^{48}$, H.-A. Gustafsson ${ }^{61}$, H. Gutbrod ${ }^{32}$, Ø. Haaland ${ }^{8}$, C. Hadjidakis ${ }^{77}$, M. Haiduc ${ }^{16}$, H. Hamagaki ${ }^{100}$, G. Hamar ${ }^{18}$, J. Hamblen ${ }^{53}$,

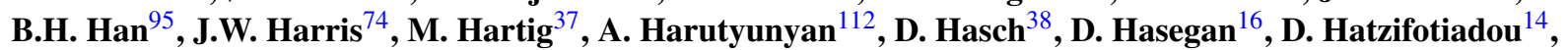
A. Hayrapetyan ${ }^{112}$, M. Heide ${ }^{72}$, M. Heinz ${ }^{74}$, H. Helstrup ${ }^{9}$, A. Herghelegiu ${ }^{17}$, C. Hernández $^{32}$, G. Herrera Corral ${ }^{64}$,

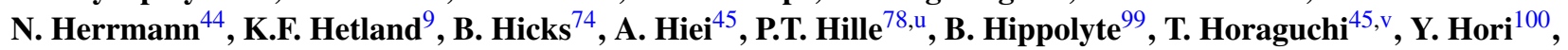
P. Hristov ${ }^{40}$, I. Hřivnáčová ${ }^{77}$, S. Hu ${ }^{7}$, S. Huber ${ }^{32}$, T.J. Humanic ${ }^{27}$, D. Hutter ${ }^{36}$, D.S. Hwang ${ }^{95}$, R. Ichou ${ }^{73}$,

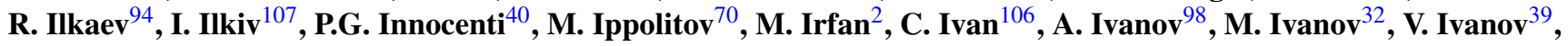
T. Iwasaki ${ }^{45}$, A. Jachołkowski ${ }^{40}$, P.M. Jacobs ${ }^{10}$, L. Jančurová ${ }^{35}$, S. Jangal ${ }^{99}$, R. Janik ${ }^{15}$, K. Jayananda ${ }^{30}$, C. Jena ${ }^{11}$, S. Jena ${ }^{71}$, L. Jirden ${ }^{40}$, G.T. Jones ${ }^{12}$, P.G. Jones ${ }^{12}$, P. Jovanović ${ }^{12}$, H. Jung ${ }^{50}$, W. Jung ${ }^{50}$, A. Jusko ${ }^{12}$, A.B. Kaidalov ${ }^{68}$, S. Kalcher ${ }^{43, c}$, P. Kaliňák ${ }^{58}$, T. Kalliokoski ${ }^{49}$, A. Kalweit ${ }^{33}$, A. Kamal ${ }^{2}$, R. Kamermans ${ }^{106}$, K. Kanaki ${ }^{8}$, E. Kang ${ }^{50}$, J.H. Kang ${ }^{96}$, J. Kapitan ${ }^{86}$, V. Kaplin ${ }^{69}$, S. Kapusta ${ }^{40}$, T. Karavicheva ${ }^{67}$, E. Karpechev ${ }^{67}$, A. Kazantsev ${ }^{70}$, U. Kebschull $^{43}$, R. Keidel ${ }^{110}$, M.M. Khan ${ }^{2}$, S.A. Khan ${ }^{55}$, A. Khanzadeev $^{39}$, Y. Kharlov ${ }^{83}$, D. Kikola ${ }^{108}$, B. Kileng 9 , D.J Kim ${ }^{49}$, D.S. Kim ${ }^{50}$, D.W. Kim ${ }^{50}$, H.N. Kim ${ }^{50}$, J. Kim ${ }^{83}$, J.H. Kim ${ }^{95}$, J.S. Kim ${ }^{50}$, M. Kim ${ }^{50}$, M. Kim ${ }^{96}$, S.H. Kim ${ }^{50}$, S. Kim ${ }^{95}$, Y. Kim ${ }^{96}$, S. Kirsch ${ }^{40}$, I. Kisel $^{43, \mathrm{e}}$, S. Kiselev ${ }^{68}$, A. Kisiel ${ }^{27, k}$, J.L. Klay ${ }^{91}$, J. Klein ${ }^{44}$, C. Klein-Bösing ${ }^{40,0}$, M. Kliemant ${ }^{37}$, A. Klovning ${ }^{8}$, A. Kluge ${ }^{40}$, S. Kniege ${ }^{37}$, K. Koch ${ }^{44}$, R. Kolevatov ${ }^{78}$, A. Kolojvari ${ }^{98}$, V. Kondratiev ${ }^{98}$, N. Kondratyeva ${ }^{69}$, A. Konevskih ${ }^{67}$, E. Kornaś $^{29}$, R. Kour ${ }^{12}$, M. Kowalski ${ }^{29}$,

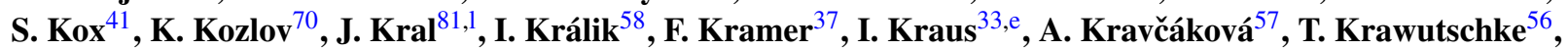
M. Krivda ${ }^{12}$, D. Krumbhorn ${ }^{44}$, M. Krus ${ }^{81}$, E. Kryshen ${ }^{39}$, M. Krzewicki ${ }^{3}$, Y. Kucheriaev ${ }^{70}$, C. Kuhn ${ }^{99}$, P.G. Kuijer ${ }^{3}$, L. Kumar ${ }^{25}$, N. Kumar ${ }^{25}$, R. Kupczak ${ }^{108}$, P. Kurashvili ${ }^{107}$, A. Kurepin ${ }^{67}$, A.N. Kurepin ${ }^{67}$, A. Kuryakin ${ }^{94}$,

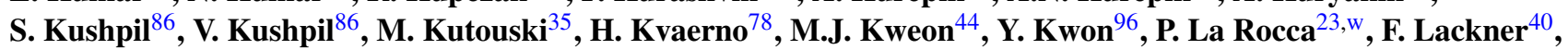
P. Ladrón de Guevara ${ }^{63}$, V. Lafage ${ }^{77}$, C. Lal $^{48}$, C. Lara $^{43}$, D.T. Larsen ${ }^{8}$, G. Laurenti ${ }^{14}$, C. Lazzeroni ${ }^{12}$,

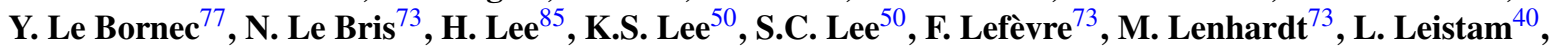
J. Lehnert ${ }^{37}$, V. Lenti ${ }^{6}$, H. León ${ }^{66}$, I. León Monzón ${ }^{31}$, H. León $\operatorname{Vargas}^{37}$, P. Lévai ${ }^{18}$, Y. Li $^{7}$, R. Lietava $^{12}$, S. Lindal $^{78}$, V. Lindenstruth ${ }^{43, c}$, C. Lippmann ${ }^{40}$, M.A. Lisa ${ }^{27}$, O. Listratenko ${ }^{51}$, L. Liu ${ }^{8}$, V. Loginov ${ }^{69}$, S. Lohn ${ }^{40}, X$. Lopez ${ }^{26}$, M. López Noriega ${ }^{77}$, R. López-Ramírez ${ }^{84}$, E. López Torres ${ }^{42}$, G. Løvhøiden ${ }^{78}$, A. Lozea Feijo Soares ${ }^{93}$, S. Lu ${ }^{7}$, M. Lunardon ${ }^{79}$, G. Luparello ${ }^{101}$, L. Luquin ${ }^{73}$, J.-R. Lutz ${ }^{99}$, M. Luvisetto ${ }^{14}$, K. Ma ${ }^{111}$, R. Ma $^{74}$, D.M. Madagodahettige-Don ${ }^{46}$, A. Maevskaya ${ }^{67}$, M. Mager $^{33, \mathrm{k}}$, A. Mahajan ${ }^{48}$, D.P. Mahapatra ${ }^{11}$, A. Maire ${ }^{99}$, I. Makhlyueva ${ }^{40}$, D. Mal'Kevich ${ }^{68}$, M. Malaev ${ }^{39}$, I. Maldonado Cervantes ${ }^{65}$, M. Malek ${ }^{77}$, T. Malkiewicz ${ }^{49}$,

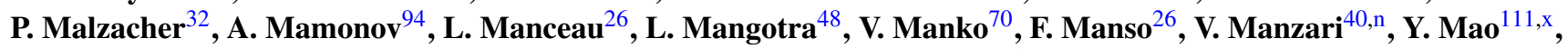
J. Mareš ${ }^{82}$, G.V. Margagliotti ${ }^{103}$, A. Margotti ${ }^{14}$, A. Marín $^{32}$, I. Martashvili ${ }^{53}$, P. Martinengo ${ }^{40}$, M.I. Martínez ${ }^{84}$, A. Martínez Davalos ${ }^{66}$, G. Martínez García ${ }^{73}$, Y. Maruyama $^{45}$, A. Marzari Chiesa ${ }^{101}$, S. Masciocchi ${ }^{32}$, M. Masera ${ }^{101}$, M. Masetti $^{13}$, A. Masoni ${ }^{20}$, L. Massacrier ${ }^{62}$, M. Mastromarco ${ }^{5}$, A. Mastroserio $^{5, k}$, Z.L. Matthews ${ }^{12}$,

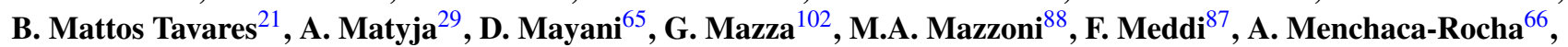
P. Mendez Lorenzo ${ }^{40}$, M. Meoni ${ }^{40}$, J. Mercado Pérez ${ }^{44}$, P. Mereu ${ }^{102}$, Y. Miake ${ }^{105}$, A. Michalon ${ }^{99}$, N. Miftakhov ${ }^{39}$, J. Milosevic ${ }^{78}$, F. Minafra ${ }^{5}$, A. Mischke ${ }^{106}$, D. Miśkowiec ${ }^{32}$, C. Mitu ${ }^{16}$, K. Mizoguchi ${ }^{45}$, J. Mlynarz ${ }^{34}$, B. Mohanty ${ }^{55}$, L. Molnar ${ }^{18, \mathrm{k}}$, M.M. Mondal ${ }^{55}$, L. Montaño Zetina ${ }^{64, \mathrm{y}}$, M. Monteno ${ }^{102}$, E. Montes $^{63}$, M. Morando ${ }^{79}$, S. Moretto ${ }^{79}$, A. Morsch ${ }^{40}$, T. Moukhanova ${ }^{70}$, V. Muccifora ${ }^{38}$, E. Mudnic $^{97}$, S. Muhuri ${ }^{55}$, H. Müller ${ }^{40}$, M.G. Munhoz ${ }^{93}$,

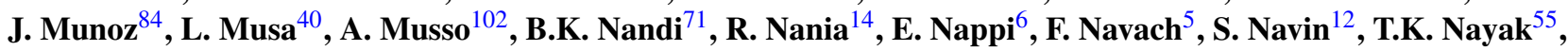
S. Nazarenko ${ }^{94}$, G. Nazarov ${ }^{94}$, A. Nedosekin ${ }^{68}$, F. Nendaz $^{62}$, J. Newby $^{60}$, A. Nianine $^{70}$, M. Nicassio ${ }^{6, \mathrm{k}}$, B.S. Nielsen ${ }^{28}$, S. Nikolaev ${ }^{70}$, V. Nikolic ${ }^{113}$, S. Nikulin ${ }^{70}$, V. Nikulin ${ }^{39}$, B.S. Nilsen ${ }^{27, z}$, M.S. Nilsson ${ }^{78}$, F. Noferini ${ }^{14}$, P. Nomokonov ${ }^{35}$, G. Nooren ${ }^{106}$, N. Novitzky ${ }^{49}$, A. Nyatha ${ }^{71}$, C. Nygaard ${ }^{28}$, A. Nyiri ${ }^{78}$, J. Nystrand $^{8}$, A. Ochirov ${ }^{98}$, G. Odyniec ${ }^{10}$, H. Oeschler ${ }^{33}$, M. Oinonen ${ }^{49}$, K. Okada ${ }^{100}$, Y. Okada ${ }^{45}$, M. Oldenburg ${ }^{40}$, J. Oleniacz $^{108}$, C. Oppedisano $^{102}$, F. Orsini ${ }^{89}$, A. Ortíz Velázquez ${ }^{65}$, G. Ortona ${ }^{101}$, C. Oskamp ${ }^{106}$, A. Oskarsson ${ }^{61}$, F. Osmic ${ }^{40}$, L. Österman ${ }^{61}$, P. Ostrowski ${ }^{108}$, I. Otterlund ${ }^{61}$, J. Otwinowski ${ }^{32}$, G. Øvrebekk ${ }^{8}$, K. Oyama ${ }^{44}$, K. Ozawa ${ }^{100}$, Y. Pachmayer ${ }^{44}$, 


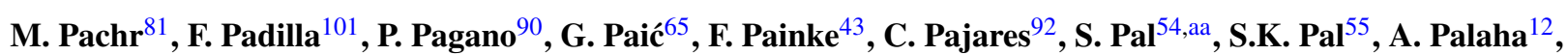
A. Palmeri ${ }^{24}$, R. Panse ${ }^{43}$, G.S. Pappalardo ${ }^{24}$, W.J. Park ${ }^{32}$, B. Pastirčák ${ }^{58}$, C. Pastore $^{6}$, V. Paticchio ${ }^{6}$, A. Pavlinov Pav $^{34}$,

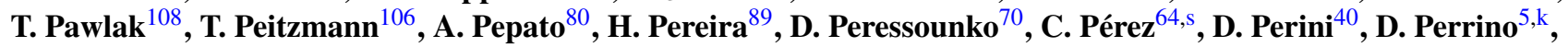
W. Peryt ${ }^{108}$, J. Peschek ${ }^{43, c}$, A. Pesci ${ }^{14}$, V. Peskov ${ }^{65, k}$, Y. Pestov ${ }^{75}$, A.J. Peters ${ }^{40}$, V. Petráček ${ }^{81}$, A. Petridis ${ }^{4, t}$,

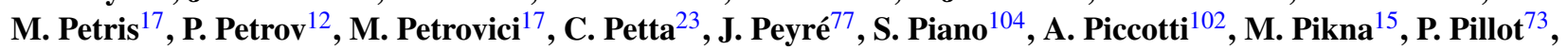

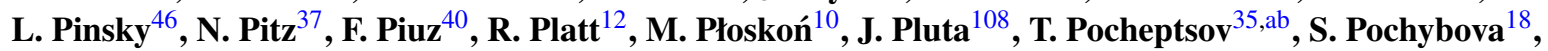
P.L.M. Podesta Lerma ${ }^{31}$, F. Poggio ${ }^{101}$, M.G. Poghosyan ${ }^{101}$, K. Polák ${ }^{82}$, B. Polichtchouk ${ }^{83}$, P. Polozov ${ }^{68}$,

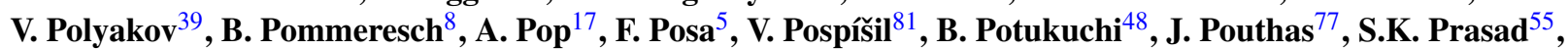
R. Preghenella ${ }^{13, \mathrm{w}}$, F. Prino ${ }^{102}$, C.A. Pruneau ${ }^{34}$, I. Pshenichnov ${ }^{67}$, G. Puddu ${ }^{19}$, P. Pujahari ${ }^{71}$, A. Pulvirenti ${ }^{23}$, A. Punin ${ }^{94}$, V. Punin ${ }^{94}$, M. Putis ${ }^{57}$, J. Putschke ${ }^{74}$, E. Quercigh $^{40}$, A. Rachevski ${ }^{104}$, A. Rademakers ${ }^{40}$, S. Radomski ${ }^{44}$, T.S. Räihä ${ }^{49}$, J. Rak ${ }^{49}$, A. Rakotozafindrabe ${ }^{89}$, L. Ramello ${ }^{1}$, A. Ramírez Reyes ${ }^{64}$, M. Rammler $^{72}$, R. Raniwala ${ }^{47}$,

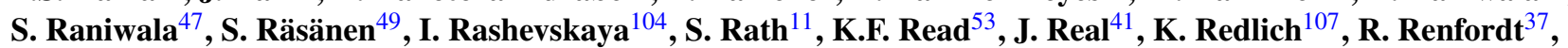

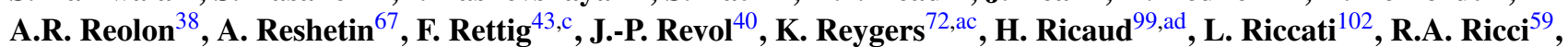

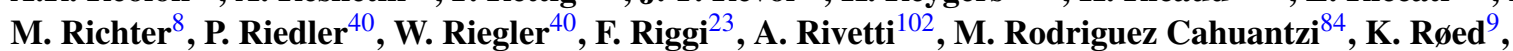

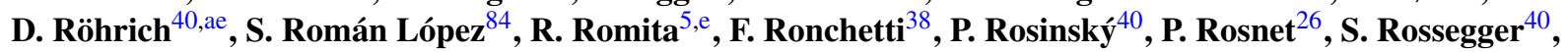
A. Rossi $^{103}$, F. Roukoutakis ${ }^{40, \text { af }}$, S. Rousseau ${ }^{77}$, C. Roy $^{73, m}$, P. Roy ${ }^{54}$, A.J. Rubio-Montero ${ }^{63}$, R. Rui ${ }^{103}$, I. Rusanov $^{44}$, G. Russo $^{90}$, E. Ryabinkin ${ }^{70}$, A. Rybicki ${ }^{29}$, S. Sadovsky ${ }^{83}$, K. Safařík ${ }^{40}$, R. Sahoo ${ }^{79}$, J. Saini ${ }^{55}$, P. Saiz ${ }^{40}$, D. Sakata ${ }^{105}$, C.A. Salgado ${ }^{92}$, R. Salgueiro Dominques da Silva $^{40}$, S. Salur ${ }^{10}$, T. Samanta ${ }^{55}$, S. Sambyal ${ }^{48}$, V. Samsonov ${ }^{39}$, L. Š́ndor ${ }^{58}$, A. Sandoval ${ }^{66}$, M. Sano ${ }^{105}$, S. Sano ${ }^{100}$, R. Santo ${ }^{72}$, R. Santoro ${ }^{5}$, J. Sarkamo ${ }^{49}$, P. Saturnini ${ }^{26}$, E. Scapparone ${ }^{14}$, F. Scarlassara ${ }^{79}$, R.P. Scharenberg ${ }^{109}$, C. Schiaua ${ }^{17}$, R. Schicker ${ }^{44}$, H. Schindler ${ }^{40}$, C. Schmidt ${ }^{32}$, H.R. Schmidt ${ }^{32}$, S. Schreiner ${ }^{40}$, S. Schuchmann ${ }^{37}$, J. Schukraft ${ }^{40, a}$, Y. Schutz ${ }^{73}$, K. Schwarz ${ }^{32}$, K. Schweda ${ }^{44}$, G. Scioli ${ }^{13}$, E. Scomparin ${ }^{102}$, G. Segato ${ }^{79}$, D. Semenov ${ }^{98}$, S. Senyukov ${ }^{1}$, J. Seo $^{50}$, S. Serci ${ }^{19}$, L. Serkin ${ }^{65}$, E. Serradilla ${ }^{63}$, A. Sevcenco ${ }^{16}$, I. Sgura ${ }^{5}$, G. Shabratova ${ }^{35}$, R. Shahoyan ${ }^{40}$, G. Sharkov ${ }^{68}$, N. Sharma ${ }^{25}$, S. Sharma ${ }^{48}$, K. Shigaki ${ }^{45}$, M. Shimomura ${ }^{105}$, K. Shtejer ${ }^{42}$, Y. Sibiriak ${ }^{70}$, M. Siciliano ${ }^{101}$, E. Sicking ${ }^{40, a g}$, E. Siddi ${ }^{20}$, T. Siemiarczuk ${ }^{107}$, A. Silenzi ${ }^{13}$, D. Silvermyr ${ }^{76}$, E. Simili ${ }^{106}$, G. Simonetti $^{5, \mathrm{k}}$, R. Singaraju ${ }^{55}$, R. Singh $^{48}$, V. Singhal ${ }^{55}$, B.C. Sinha ${ }^{55}$, T. Sinha ${ }^{54}$, B. Sitar ${ }^{15}$, M. Sitta ${ }^{1}$, T.B. Skaali ${ }^{78}$, K. Skjerdal ${ }^{8}$, R. Smakal ${ }^{81}$, N. Smirnov ${ }^{74}$, R. Snellings ${ }^{3}$, H. Snow ${ }^{12}$, C. Søgaard ${ }^{28}$, O. Sokolov ${ }^{65}$, A. Soloviev ${ }^{83}$, H.K. Soltveit ${ }^{44}$, R. Soltz ${ }^{60}$, W. Sommer ${ }^{37}$, C.W. Son ${ }^{85}$, H.S. Son ${ }^{95}$, M. Song ${ }^{96}$, C. Soos $^{40}$, F. Soramel ${ }^{79}$, D. Soyk ${ }^{32}$, M. Spyropoulou-Stassinaki ${ }^{4}$, B.K. Srivastava ${ }^{109}$,

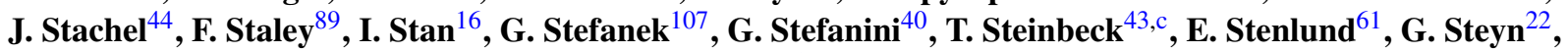
D. Stocco ${ }^{101, \text { ah }}$, R. Stock ${ }^{37}$, P. Stolpovsky ${ }^{83}$, P. Strmen ${ }^{15}$, A.A.P. Suaide ${ }^{93}$, M.A. Subieta Vásquez ${ }^{101}$, T. Sugitate ${ }^{45}$, C. Suire ${ }^{77}$, M. Sumbera ${ }^{86}$, T. Susa ${ }^{113}$, D. Swoboda ${ }^{40}$, T.J.M. Symons ${ }^{10}$, A. Szanto de Toledo ${ }^{93}$, I. Szarka ${ }^{15}$, A. Szostak ${ }^{20}$, M. Szuba ${ }^{108}$, M. Tadel ${ }^{40}$, C. Tagridis ${ }^{4}$, A. Takahara ${ }^{100}$, J. Takahashi ${ }^{21}$, R. Tanabe ${ }^{105}$, J.D. Tapia Takaki ${ }^{77}$, H. Taureg ${ }^{40}$, A. Tauro ${ }^{40}$, M. Tavlet ${ }^{40}$, G. Tejeda Muñoz ${ }^{84}$, A. Telesca ${ }^{40}$, C. Terrevoli ${ }^{5}$, J. Thäder ${ }^{43, c}$, R. Tieulent ${ }^{62}$, D. Tlusty ${ }^{81}$, A. Toia ${ }^{40}$, T. Tolyhy ${ }^{18}$, C. Torcato de Matos $^{40}$, H. Torii ${ }^{45}$, G. Torralba ${ }^{43}$,

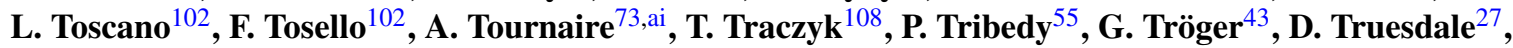
W.H. Trzaska ${ }^{49}$, G. Tsiledakis ${ }^{44}$, E. Tsilis ${ }^{4}$, T. Tsuji ${ }^{100}$, A. Tumkin $^{94}$, R. Turrisi ${ }^{80}$, A. Turvey $^{30}$, T.S. Tveter ${ }^{78}$, H. Tydesjö ${ }^{40}$, K. Tywoniuk ${ }^{78}$, J. Ulery ${ }^{37}$, K. Ullaland ${ }^{8}$, A. Uras $^{19}{ }^{19}$ J. Urbán ${ }^{57}$, G.M. Urciuoli ${ }^{88}$, G.L. Usai ${ }^{19}$, A. Vacchi ${ }^{104}$, M. Vala ${ }^{35, j}$, L. Valencia Palomo ${ }^{66}$, S. Vallero ${ }^{44}$, A. van den Brink ${ }^{106}$, N. van der Kolk ${ }^{3}$,

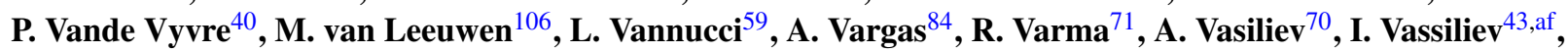

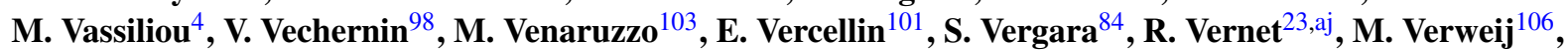

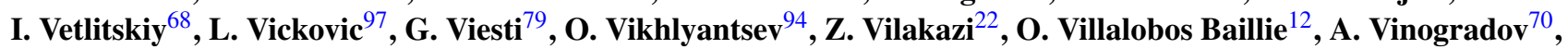
L. Vinogradov ${ }^{98}$, Y. Vinogradov ${ }^{94}$, T. Virgili ${ }^{90}$, Y.P. Viyogi ${ }^{11, a k}$, A. Vodopianov ${ }^{35}$, K. Voloshin ${ }^{68}$, S. Voloshin ${ }^{34}$, G. Volpe ${ }^{5}$, B. von Haller ${ }^{40}$, D. Vranic ${ }^{32}$, J. Vrláková ${ }^{57}$, B. Vulpescu ${ }^{26}$, B. Wagner ${ }^{8}$, V. Wagner $^{81}$, L. Wallet ${ }^{40}$, R. Wan ${ }^{111, x}$, D. Wang ${ }^{111}$, Y. Wang ${ }^{44}$, Y. Wang ${ }^{111}$, K. Watanabe ${ }^{105}$, Q. Wen ${ }^{7}$, J. Wessels ${ }^{72}$, J. Wiechula ${ }^{44}$, J. Wikne $^{78}$, A. Wilk ${ }^{72}$, G. Wilk ${ }^{107}$, M.C.S. Williams ${ }^{14}$, N. Willis ${ }^{77}$, B. Windelband ${ }^{44}$, C. Xu ${ }^{111}$, C. Yang ${ }^{111}$, H. Yang ${ }^{44}$,

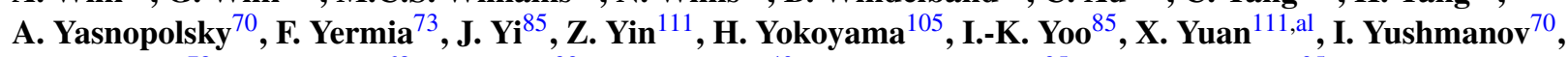
E. Zabrodin ${ }^{78}$, B. Zagreev ${ }^{68}$, A. Zalite ${ }^{39}$, C. Zampolli ${ }^{40, a m}$, Yu. Zanevsky ${ }^{35}$, S. Zaporozhets ${ }^{35}$, A. Zarochentsev ${ }^{98}$, P. Závada ${ }^{82}$, H. Zbroszczyk ${ }^{108}$, P. Zelnicek ${ }^{43}$, A. Zenin ${ }^{83}$, A. Zepeda ${ }^{64}$, I. Zgura $^{16}$, M. Zhalov ${ }^{39}$, X. Zhang ${ }^{111, b}$, D. Zhou ${ }^{111}$, S. Zhou ${ }^{7}$, S. Zhou ${ }^{7}$, J. Zhu ${ }^{111}$, A. Zichichi ${ }^{13, w}$, A. Zinchenko ${ }^{35}$, G. Zinovjev ${ }^{52}$, M. Zinovjev ${ }^{52}$, Y. Zoccarato ${ }^{62}$, V. Zycháček ${ }^{81}$

\footnotetext{
${ }^{1}$ Dipartimento di Scienze e Tecnologie Avanzate dell'Università del Piemonte Orientale and Gruppo Collegato INFN, Alessandria, Italy

${ }^{2}$ Department of Physics, Aligarh Muslim University, Aligarh, India

${ }^{3}$ National Institute for Nuclear and High Energy Physics (NIKHEF), Amsterdam, The Netherlands
} 
${ }^{4}$ Physics Department, University of Athens, Athens, Greece

${ }^{5}$ Dipartimento Interateneo di Fisica 'M. Merlin' and Sezione INFN, Bari, Italy

${ }^{6}$ Sezione INFN, Bari, Italy

${ }^{7}$ China Institute of Atomic Energy, Beijing, China

${ }^{8}$ Department of Physics and Technology, University of Bergen, Bergen, Norway

${ }^{9}$ Faculty of Engineering, Bergen University College, Bergen, Norway

${ }^{10}$ Lawrence Berkeley National Laboratory, Berkeley, CA, USA

${ }^{11}$ Institute of Physics, Bhubaneswar, India

${ }^{12}$ School of Physics and Astronomy, University of Birmingham, Birmingham, UK

${ }^{13}$ Dipartimento di Fisica dell'Università and Sezione INFN, Bologna, Italy

${ }^{14}$ Sezione INFN, Bologna, Italy

${ }^{15}$ Faculty of Mathematics, Physics and Informatics, Comenius University, Bratislava, Slovakia

${ }^{16}$ Institute of Space Sciences (ISS), Bucharest, Romania

${ }^{17}$ National Institute for Physics and Nuclear Engineering, Bucharest, Romania

${ }^{18}$ KFKI Research Institute for Particle and Nuclear Physics, Hungarian Academy of Sciences, Budapest, Hungary

${ }^{19}$ Dipartimento di Fisica dell'Università and Sezione INFN, Cagliari, Italy

${ }^{20}$ Sezione INFN, Cagliari, Italy

${ }^{21}$ Universidade Estadual de Campinas (UNICAMP), Campinas, Brazil

${ }^{22}$ Physics Department, University of Cape Town, iThemba Laboratories, Cape Town, South Africa

${ }^{23}$ Dipartimento di Fisica e Astronomia dell'Università and Sezione INFN, Catania, Italy

${ }^{24}$ Sezione INFN, Catania, Italy

${ }^{25}$ Physics Department, Panjab University, Chandigarh, India

${ }^{26}$ Laboratoire de Physique Corpusculaire (LPC), Clermont Université, Université Blaise Pascal, CNRS-IN2P3, Clermont-Ferrand, France

${ }^{27}$ Department of Physics, Ohio State University, Columbus, OH, USA

${ }^{28}$ Niels Bohr Institute, University of Copenhagen, Copenhagen, Denmark

${ }^{29}$ The Henryk Niewodniczanski Institute of Nuclear Physics, Polish Academy of Sciences, Cracow, Poland

${ }^{30}$ Physics Department, Creighton University, Omaha, NE, USA

${ }^{31}$ Universidad Autónoma de Sinaloa, Culiacán, Mexico

${ }^{32}$ ExtreMe Matter Institute EMMI, GSI Helmholtzzentrum für Schwerionenforschung, Darmstadt, Germany

${ }^{33}$ Institut für Kernphysik, Technische Universität Darmstadt, Darmstadt, Germany

${ }^{34}$ Wayne State University, Detroit, MI, USA

${ }^{35}$ Joint Institute for Nuclear Research (JINR), Dubna, Russia

${ }^{36}$ Frankfurt Institute for Advanced Studies, Johann Wolfgang Goethe-Universität Frankfurt, Frankfurt, Germany

${ }^{37}$ Institut für Kernphysik, Johann Wolfgang Goethe-Universität Frankfurt, Frankfurt, Germany

${ }^{38}$ Laboratori Nazionali di Frascati, INFN, Frascati, Italy

${ }^{39}$ Petersburg Nuclear Physics Institute, Gatchina, Russia

${ }^{40}$ European Organization for Nuclear Research (CERN), Geneva, Switzerland

${ }^{41}$ Laboratoire de Physique Subatomique et de Cosmologie (LPSC), Université Joseph Fourier, CNRS-IN2P3, Institut Polytechnique de Grenoble, Grenoble, France

${ }^{42}$ Centro de Aplicaciones Tecnológicas y Desarrollo Nuclear (CEADEN), Havana, Cuba

${ }^{43}$ Kirchhoff-Institut für Physik, Ruprecht-Karls-Universität Heidelberg, Heidelberg, Germany

${ }^{44}$ Physikalisches Institut, Ruprecht-Karls-Universität Heidelberg, Heidelberg, Germany

${ }^{45}$ Hiroshima University, Hiroshima, Japan

${ }^{46}$ University of Houston, Houston, TX, USA

${ }^{47}$ Physics Department, University of Rajasthan, Jaipur, India

${ }^{48}$ Physics Department, University of Jammu, Jammu, India

${ }^{49}$ Helsinki Institute of Physics (HIP) and University of Jyväskylä, Jyväskylä, Finland

${ }^{50}$ Kangnung National University, Kangnung, South Korea

${ }^{51}$ Scientific Research Technological Institute of Instrument Engineering, Kharkov, Ukraine

${ }_{53}^{52}$ Bogolyubov Institute for Theoretical Physics, Kiev, Ukraine

${ }^{53}$ University of Tennessee, Knoxville, TN, USA

${ }^{54}$ Saha Institute of Nuclear Physics, Kolkata, India

${ }^{55}$ Variable Energy Cyclotron Centre, Kolkata, India

${ }^{56}$ Fachhochschule Köln, Köln, Germany

${ }^{57}$ Faculty of Science, P.J. Šafárik University, Košice, Slovakia

${ }^{58}$ Institute of Experimental Physics, Slovak Academy of Sciences, Košice, Slovakia

${ }^{59}$ Laboratori Nazionali di Legnaro, INFN, Legnaro, Italy

${ }^{60}$ Lawrence Livermore National Laboratory, Livermore, CA, USA

${ }^{61}$ Division of Experimental High Energy Physics, University of Lund, Lund, Sweden

${ }^{62}$ Université de Lyon 1, CNRS/IN2P3, Institut de Physique Nucléaire de Lyon, Lyon, France

${ }^{63}$ Centro de Investigaciones Energéticas Medioambientales y Tecnológicas (CIEMAT), Madrid, Spain

${ }^{64}$ Centro de Investigación y de Estudios Avanzados (CINVESTAV), Mexico City and Mérida, Mexico

${ }^{65}$ Instituto de Ciencias Nucleares, Universidad Nacional Autónoma de México, Mexico City, Mexico

${ }^{66}$ Instituto de Física, Universidad Nacional Autónoma de México, Mexico City, Mexico

${ }^{67}$ Institute for Nuclear Research, Academy of Sciences, Moscow, Russia 
${ }^{68}$ Institute for Theoretical and Experimental Physics, Moscow, Russia

${ }^{69}$ Moscow Engineering Physics Institute, Moscow, Russia

${ }^{70}$ Russian Research Centre Kurchatov Institute, Moscow, Russia

${ }^{71}$ Indian Institute of Technology, Mumbai, India

${ }^{72}$ Institut für Kernphysik, Westfälische Wilhelms-Universität Münster, Münster, Germany

${ }^{73}$ SUBATECH, Ecole des Mines de Nantes, Université de Nantes, CNRS-IN2P3, Nantes, France

${ }^{74}$ Yale University, New Haven, CT, USA

${ }^{75}$ Budker Institute for Nuclear Physics, Novosibirsk, Russia

${ }_{77}^{76}$ Oak Ridge National Laboratory, Oak Ridge, TN, USA

${ }^{77}$ Institut de Physique Nucléaire d'Orsay (IPNO), Université Paris-Sud, CNRS-IN2P3, Orsay, France

${ }^{78}$ Department of Physics, University of Oslo, Oslo, Norway

${ }^{79}$ Dipartimento di Fisica dell'Università and Sezione INFN, Padova, Italy

${ }^{80}$ Sezione INFN, Padova, Italy

${ }^{81}$ Faculty of Nuclear Sciences and Physical Engineering, Czech Technical University in Prague, Prague, Czech Republic

${ }^{82}$ Institute of Physics, Academy of Sciences of the Czech Republic, Prague, Czech Republic

${ }^{83}$ Institute for High Energy Physics, Protvino, Russia

${ }^{84}$ Benemérita Universidad Autónoma de Puebla, Puebla, Mexico

${ }^{85}$ Pusan National University, Pusan, South Korea

${ }^{86}$ Nuclear Physics Institute, Academy of Sciences of the Czech Republic, Řež u Prahy, Czech Republic

${ }^{87}$ Dipartimento di Fisica dell'Università 'La Sapienza' and Sezione INFN, Rome, Italy

${ }^{88}$ Sezione INFN, Rome, Italy

${ }^{89}$ Commissariat à l'Energie Atomique, IRFU, Saclay, France

${ }^{90}$ Dipartimento di Fisica 'E.R. Caianiello' dell’Università and Sezione INFN, Salerno, Italy

${ }^{91}$ California Polytechnic State University, San Luis Obispo, CA, USA

${ }^{92}$ Departamento de Física de Partículas and IGFAE, Universidad de Santiago de Compostela, Santiago de Compostela, Spain

${ }^{93}$ Universidade de São Paulo (USP), São Paulo, Brazil

${ }^{94}$ Russian Federal Nuclear Center (VNIIEF), Sarov, Russia

${ }^{95}$ Department of Physics, Sejong University, Seoul, South Korea

${ }^{96}$ Yonsei University, Seoul, South Korea

${ }^{97}$ Technical University of Split FESB, Split, Croatia

${ }^{98}$ V. Fock Institute for Physics, St. Petersburg State University, St. Petersburg, Russia

${ }^{99}$ Institut Pluridisciplinaire Hubert Curien (IPHC), Université de Strasbourg, CNRS-IN2P3, Strasbourg, France

${ }^{100}$ University of Tokyo, Tokyo, Japan

${ }^{101}$ Dipartimento di Fisica Sperimentale dell'Università and Sezione INFN, Turin, Italy

${ }^{102}$ Sezione INFN, Turin, Italy

${ }^{103}$ Dipartimento di Fisica dell'Università and Sezione INFN, Trieste, Italy

${ }^{104}$ Sezione INFN, Trieste, Italy

${ }^{105}$ University of Tsukuba, Tsukuba, Japan

${ }^{106}$ Institute for Subatomic Physics, Utrecht University, Utrecht, The Netherlands

${ }^{107}$ Soltan Institute for Nuclear Studies, Warsaw, Poland

${ }^{108}$ Warsaw University of Technology, Warsaw, Poland

${ }^{109}$ Purdue University, West Lafayette, IN, USA

${ }^{110}$ Zentrum für Technologietransfer und Telekommunikation (ZTT), Fachhochschule Worms, Worms, Germany

${ }^{111}$ Hua-Zhong Normal University, Wuhan, China

${ }^{112}$ Yerevan Physics Institute, Yerevan, Armenia

${ }^{113}$ Rudjer Bošković Institute, Zagreb, Croatia

Received: 28 November 2009 / Revised: 1 December 2009 / Published online: 11 December 2009

(C) CERN 2009. This article is published with open access at Springerlink.com

a e-mail: Jurgen.Schukraft@cern.ch

${ }^{\mathrm{b}}$ Also at Laboratoire de Physique Corpusculaire (LPC), Clermont Université, Université Blaise Pascal, CNRS-IN2P3, Clermont-Ferrand, France.

${ }^{\mathrm{c}}$ Also at Frankfurt Institute for Advanced Studies, Johann Wolfgang Goethe-Universität Frankfurt, Frankfurt, Germany.

${ }^{\mathrm{d}}$ Now at Sezione INFN, Padova, Italy.

eNow at ExtreMe Matter Institute EMMI, GSI Helmholtzzentrum für Schwerionenforschung, Darmstadt, Germany.

${ }^{\mathrm{f}}$ Now at Institut für Kernphysik, Johann Wolfgang Goethe-Universität Frankfurt, Frankfurt, Germany.
${ }^{\mathrm{g}}$ Now at Physics Department, University of Cape Town, iThemba Laboratories, Cape Town, South Africa.

${ }^{\mathrm{h}}$ Now at National Institute for Physics and Nuclear Engineering, Bucharest, Romania.

${ }^{\mathrm{i}}$ Also at University of Houston, Houston, TX, USA

jNow at Faculty of Science, P.J. Šafárik University, Košice, Slovakia.

${ }^{k}$ Now at European Organization for Nuclear Research (CERN), Geneva, Switzerland.

${ }^{1}$ Now at Helsinki Institute of Physics (HIP) and University of Jyväskylä, Jyväskylä, Finland. 
Abstract On 23rd November 2009, during the early commissioning of the CERN Large Hadron Collider (LHC), two counter-rotating proton bunches were circulated for the first time concurrently in the machine, at the LHC injection energy of $450 \mathrm{GeV}$ per beam. Although the proton intensity was very low, with only one pilot bunch per beam, and no systematic attempt was made to optimize the collision optics, all LHC experiments reported a number of collision candidates. In the ALICE experiment, the collision region was centred very well in both the longitudinal and trans-

${ }^{m}$ Now at Institut Pluridisciplinaire Hubert Curien (IPHC), Université de Strasbourg, CNRS-IN2P3, Strasbourg, France.

${ }^{\mathrm{n}}$ Now at Sezione INFN, Bari, Italy.

${ }^{\circ}$ Now at Institut für Kernphysik, Westfälische Wilhelms-Universität Münster, Münster, Germany.

pNow at University of Technology and Austrian Academy of Sciences, Vienna, Austria.

${ }^{\mathrm{q}}$ Also at Lawrence Livermore National Laboratory, Livermore, CA, USA.

${ }^{\mathrm{r}}$ Also at European Organization for Nuclear Research (CERN), Geneva, Switzerland.

${ }^{\mathrm{s}}$ Now at Sección Física, Departamento de Ciencias, Pontificia Universidad Católica del Perú, Lima, Peru.

${ }^{\mathrm{t}}$ Deceased.

"Now at Yale University, New Haven, CT, USA.

${ }^{\mathrm{v}}$ Now at University of Tsukuba, Tsukuba, Japan.

w Also at Centro Fermi-Centro Studi e Ricerche e Museo Storico della Fisica "Enrico Fermi”, Rome, Italy.

${ }^{x}$ Also at Laboratoire de Physique Subatomique et de Cosmologie (LPSC), Université Joseph Fourier, CNRS-IN2P3, Institut Polytechnique de Grenoble, Grenoble, France.

${ }^{y}$ Now at Dipartimento di Fisica Sperimentale dell'Università and Sezione INFN, Turin, Italy.

${ }^{z}$ Now at Physics Department, Creighton University, Omaha, NE, USA.

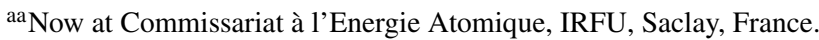

${ }^{\mathrm{ab}}$ Also at Department of Physics, University of Oslo, Oslo, Norway.

${ }^{a c}$ Now at Physikalisches Institut, Ruprecht-Karls-Universität Heidelberg, Heidelberg, Germany.

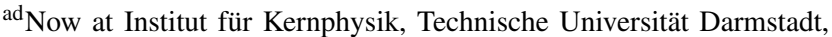
Darmstadt, Germany.

${ }^{a e}$ Now at Department of Physics and Technology, University of Bergen, Bergen, Norway.

${ }^{a f}$ Now at Physics Department, University of Athens, Athens, Greece.

${ }^{a g}$ Also at Institut für Kernphysik, Westfälische Wilhelms-Universität Münster, Münster, Germany.

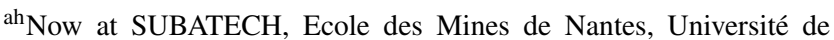
Nantes, CNRS-IN2P3, Nantes, France.

${ }^{a i}$ Now at Université de Lyon 1, CNRS/IN2P3, Institut de Physique Nucléaire de Lyon, Lyon, France.

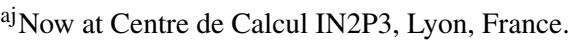

${ }^{\mathrm{ak}}$ Now at Variable Energy Cyclotron Centre, Kolkata, India.

${ }^{\text {al } A l s o ~ a t ~ D i p a r t i m e n t o ~ d i ~ F i s i c a ~ d e l l ' U n i v e r s i t a ̀ ~ a n d ~ S e z i o n e ~ I N F N, ~}$

Padova, Italy.

am Also at Sezione INFN, Bologna, Italy. verse directions and 284 events were recorded in coincidence with the two passing proton bunches. The events were immediately reconstructed and analyzed both online and offline. We have used these events to measure the pseudorapidity density of charged primary particles in the central region. In the range $|\eta|<0.5$, we obtain $\mathrm{d} N_{\mathrm{ch}} / \mathrm{d} \eta=$ $3.10 \pm 0.13$ (stat.) \pm 0.22 (syst.) for all inelastic interactions, and $\mathrm{d} N_{\mathrm{ch}} / \mathrm{d} \eta=3.51 \pm 0.15$ (stat.) \pm 0.25 (syst.) for nonsingle diffractive interactions. These results are consistent with previous measurements in proton-antiproton interactions at the same centre-of-mass energy at the CERN Spp $\bar{S}$ collider. They also illustrate the excellent functioning and rapid progress of the LHC accelerator, and of both the hardware and software of the ALICE experiment, in this early start-up phase.

\section{Introduction}

The very first proton-proton collisions at Point 2 of the CERN Large Hadron Collider (LHC) [1] occurred in the afternoon of 23rd November 2009, at a centre-of-mass energy $\sqrt{s}=900 \mathrm{GeV}$, during the commissioning of the accelerator. This publication, based on 284 events recorded in the ALICE detector [2] on that day, describes a determination of the pseudorapidity density of charged primary particles ${ }^{1}$ $\mathrm{d} N_{\mathrm{ch}} / \mathrm{d} \eta(\eta \equiv-\ln \tan \theta / 2$, where $\theta$ is the polar angle with respect to the beam line) in the central pseudorapidity region. The purpose of this study is to compare with previous measurements for proton-antiproton $(\mathrm{p} \overline{\mathrm{p}})$ collisions at the same energy [3], and to establish a reference for comparison with forthcoming measurements at higher LHC energies.

The event sample collected with our trigger contains three different classes of inelastic interactions, i.e. collisions where new particles are produced: non-diffractive, singlediffractive, and double-diffractive. ${ }^{2}$ Experimentally we cannot distinguish between these classes, which, however, are selected by our trigger with different efficiencies. ${ }^{3}$

\footnotetext{
${ }^{1}$ Here, primary particles are defined as prompt particles produced in the collision and all decay products, except products from weak decays of strange particles such as $\mathrm{K}_{\mathrm{s}}^{0}$ and $\Lambda$.

${ }^{2}$ Inelastic pp collisions are usually divided into these classes depending on the fate of the interacting protons. If one (both) incoming beam particle(s) are excited into a high-mass state, the process is called single (double) diffraction; otherwise the events are classified as nondiffractive. Particles emitted in diffractive reactions are usually found at rapidities close to that of the parent proton.

${ }^{3}$ We estimate the trigger efficiency for each class using the process-type information provided by Monte Carlo generators; the values vary by up to a factor of two between classes and are listed in Sect. 3. The relative abundance of each class is taken from published data (see text).
} 
In order to compare our data with those of other experiments, we provide the result with two different normalizations: the first one (INEL) corresponds to the sum of all inelastic interactions and corrects the trigger bias individually for all event classes, by weighting them, each with its own estimated trigger efficiency and abundance. The second normalization (non-single-diffractive or NSD) applies this correction for non-diffractive and double-diffractive processes only, while removing, on average, the single-diffractive contribution.

Multiparticle production is rather successfully described by phenomenological models with Pomeron exchange, which dominates at high energies [4-11]. These models relate the energy dependence of the total cross section to that of the multiplicity production using a small number of parameters, and are the basis for several Monte Carlo event generators describing soft hadron collisions (see for example [12-16]). According to these models, it is expected that the charged-particle density increases by a factor 1.7 and 1.9 when raising the LHC centre-of-mass energy from $900 \mathrm{GeV}$ to 7 and $14 \mathrm{TeV}$ respectively (i.e. intermediate and nominal LHC energies). The difference in charged-particle densities between $\mathrm{p} \overline{\mathrm{p}}$ and $\mathrm{pp}$ interactions is predicted to decrease as $1 / \sqrt{s}$ at high energies [17]. This difference was last measured at the CERN ISR to be in the range 1.5$3 \%[18,19]$ at $\sqrt{s}=53 \mathrm{GeV}$. Extrapolating these values to $\sqrt{s}=900 \mathrm{GeV}$, one obtains a very small difference of about $0.1-0.2 \%$. Therefore, we will compare our measurement to existing $\mathrm{p} \overline{\mathrm{p}}$ data and also to different Monte Carlo models.

This article is organized as follows: Sect. 2 describes the experimental conditions during data taking; the main features of the ALICE detector subsystems used for this analysis are described in Sect. 3; Sect. 4 is dedicated to the event selection and data analysis; the results are discussed in Sect. 5 and Sect. 6 contains the conclusion.

\section{LHC and the run conditions}

The LHC, built at CERN in the circular tunnel of $27 \mathrm{~km}$ circumference previously used by the Large Electron-Positron collider (LEP), will provide the highest energy ever explored with particle accelerators. It is designed to collide two counter-rotating beams of protons or heavy ions. The nominal centre-of-mass energy for proton-proton collisions is $14 \mathrm{TeV}$. However, collisions can be obtained down to $\sqrt{s}=900 \mathrm{GeV}$, which corresponds to the beam injection energy.

The results from the first proton-proton collisions presented here were obtained during the early commissioning phase of the LHC, when two proton bunches were circulating for the first time concurrently in the machine. The bunches used were the so-called "pilot bunches": low intensity bunches used during machine commissioning, with a few $10^{9}$ protons per bunch. The two beams were brought into the nominal position for collisions without a specific attempt to maximize the interaction rate. The nominal r.m.s. size of LHC beams at injection energy is about $300 \mu \mathrm{m}$ in the transverse direction and $10.5 \mathrm{~cm}$ in the longitudinal ( $z$-axis) direction. However, at this early stage, the beam parameters can deviate from these nominal values; they were not measured for the fill used in this analysis. For the previous fill, for which the longitudinal size was measured, it was found to be shorter, with an r.m.s. of about $8 \mathrm{~cm}$. Assuming Gaussian beam profiles, the luminous region should be smaller than the beam size by a factor of $\sqrt{2}$ in all directions.

Shortly after circulating beams were established, the ALICE data acquisition system [20,21] started collecting events with a trigger based on the Silicon Pixel Detector (SPD), requiring two or more hits in the SPD in coincidence with the passage of the two colliding bunches as inferred from beam pickup detectors. As a precaution, only a small subset of the detector subsystems, including the silicon tracking detectors and the scintillator trigger counters, was turned on, in order to assess the beam conditions provided by the LHC.

The trigger rate was measured just before collisions with the same trigger conditions. Without beams we measured a rate of $3 \times 10^{-4} \mathrm{~Hz}$ (in coincidence with one bunch crossing interval per orbit). In coincidence with the passage of the bunch of one circulating beam the rate was $0.006 \mathrm{~Hz}$. As soon as the second beam was injected in the accelerator, the event rate increased significantly, to $0.11 \mathrm{~Hz}$. The first event that was analyzed and displayed in the counting room by the offline reconstruction software AliRoot [22] running in online mode is shown in Fig. 1. This marked symbolically the keenly anticipated start of the physics exploitation of the ALICE experiment. ${ }^{4}$ The online reconstruction software implemented in the High-Level Trigger (HLT) computer farm [23] also analyzed the events in real time and calculated the vertex position of the collected events, shown in Fig. 2. The distributions are very narrow in the transverse plane (sub-millimetre, including contributions from detector resolution and residual misalignment), of about the expected size in the longitudinal direction and well positioned with respect to the nominal centre of the ALICE detector. This provided immediate evidence that a substantial fraction of the events corresponded to collisions between the protons of the two counter-rotating beams.

After 43 minutes, the two beams were dumped in order to proceed with the LHC commissioning programme. In total, 284 events were triggered and recorded during this short, but important, first run of the ALICE experiment with colliding beams.

\footnotetext{
${ }^{4}$ The event display started shortly after data taking and therefore missed the first few events.
} 


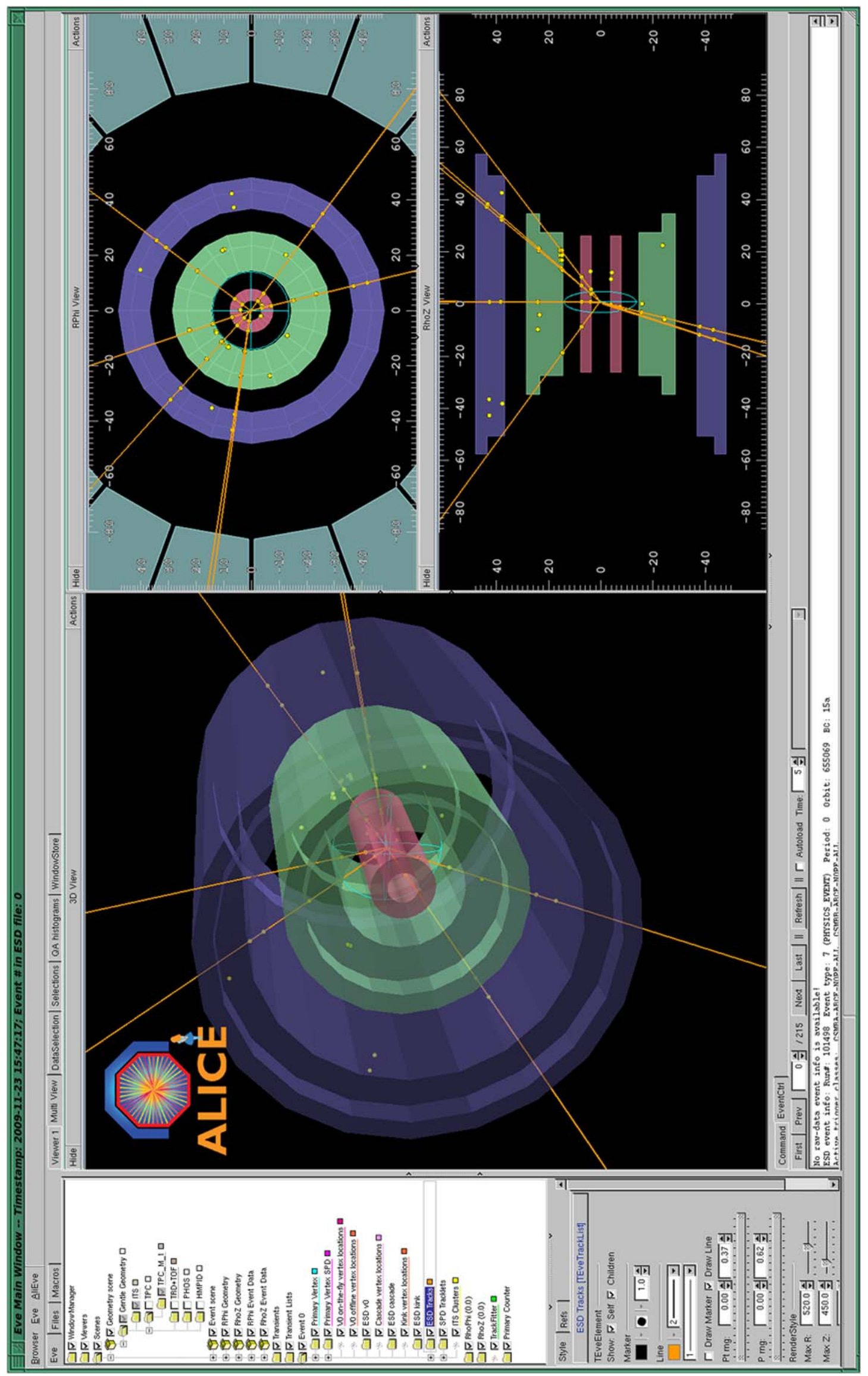

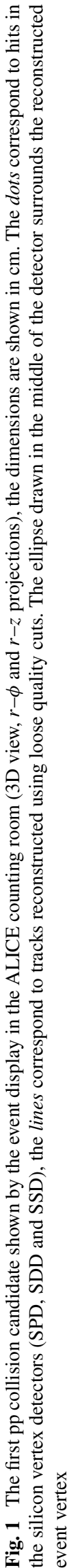




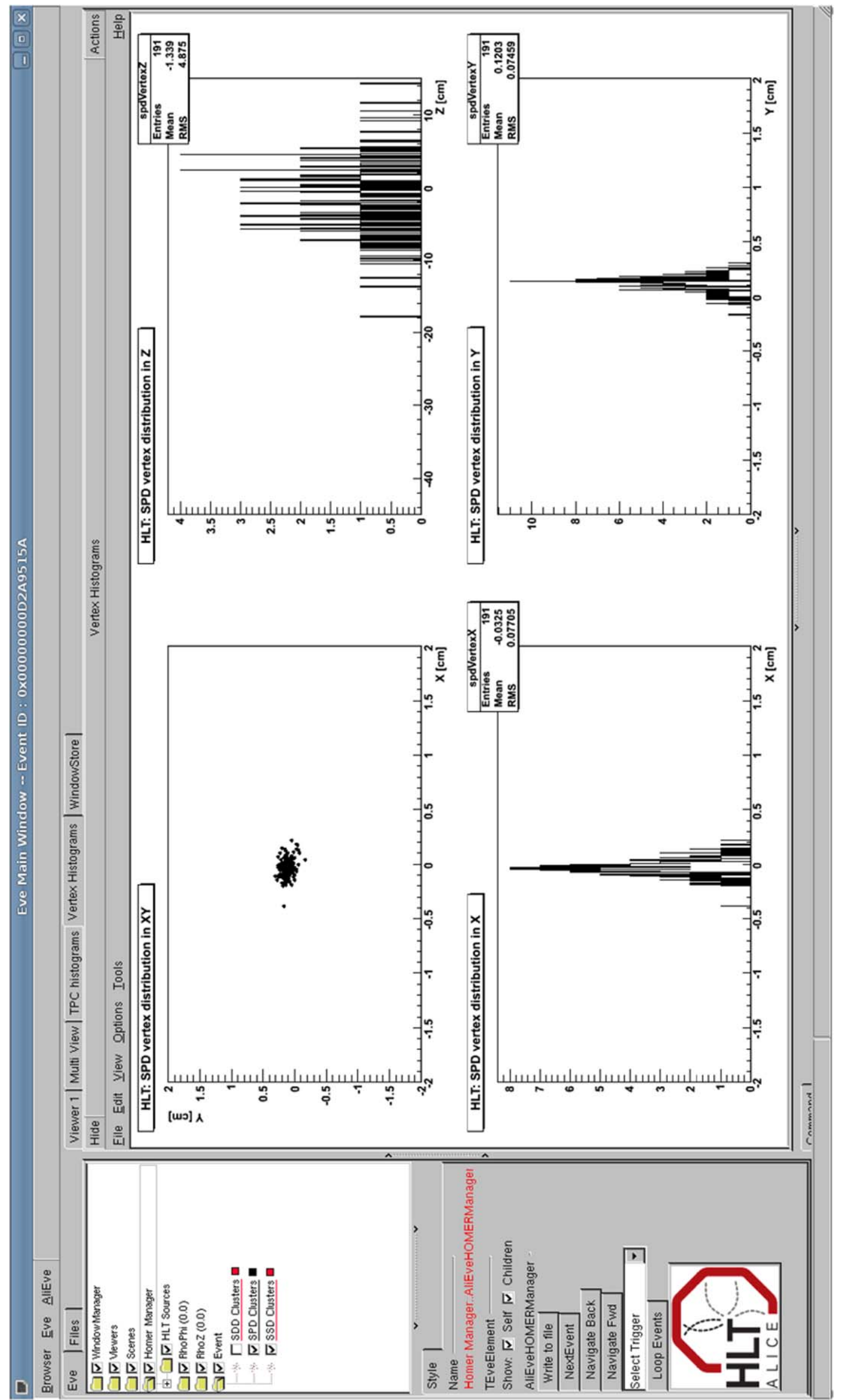

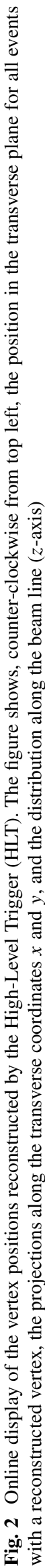




\section{The ALICE experiment}

ALICE, designed as the dedicated heavy-ion experiment at the LHC, also has excellent performance for proton-proton interactions [24, 25]. The experiment consists of a large number of detector subsystems [2] inside a solenoidal magnet $(B=0.5 \mathrm{~T})$. The magnet was off during this run.

During the several months of running with cosmic rays in 2008 and 2009, all of the ALICE detector subsystems were extensively commissioned, calibrated and used for data taking [26-30]. Data were collected for an initial alignment of the parts of the detector that had sufficient exposure to the mostly vertical cosmic ray flux. Data were also taken during various LHC injection tests to perform timing measurements and other calibrations.

Collisions take place at the centre of the ALICE detector, inside a beryllium vacuum beam pipe $(3 \mathrm{~cm}$ in radius and $800 \mu \mathrm{m}$ thick). The tracking system in the ALICE central barrel covers the full azimuthal range in the pseudorapidity window $|\eta|<0.9$. It has been designed to cope with the highest charged-particle densities expected in central $\mathrm{Pb}-\mathrm{Pb}$ collisions. The following four detector subsystems were active during data taking and were used in this analysis:

- The Silicon Pixel Detector (SPD) consists of two cylindrical layers with radii of 3.9 and $7.6 \mathrm{~cm}$ and has about 9.8 million pixels of size $50 \times 425 \mu \mathrm{m}^{2}$. It covers the pseudorapidity ranges $|\eta|<2$ and $|\eta|<1.4$ for the inner and outer layers respectively, for particles originating at the centre of the detector. The effective $\eta$-acceptance is larger due to the longitudinal spread of the position of the interaction vertex. The detector is read out by customdesigned ASICs bump-bonded directly on silicon ladders. Each chip contains 8192 channels and also provides a fast trigger signal if at least one of its pixels is hit. The trigger signals from all 1200 chips are then combined in a programmable logic unit which provides a level-0 trigger signal to the central trigger processor. The total thickness of the SPD amounts to about $2.3 \%$ of a radiation length. About $83 \%$ of the channels were operational for particle detection and $77 \%$ of the chips were used in the trigger logic. The SPD was aligned using cosmic-ray tracks collected during 2008 [31], and the residual misalignment was estimated to be below $10 \mu \mathrm{m}$ for the modules well covered by mostly vertical tracks. The modules on the sides are likely to be affected by larger residual misalignment.

- The Silicon Drift Detector (SDD) consists of two cylindrical layers at radii of 15.0 and $23.9 \mathrm{~cm}$ and covers the region $|\eta|<0.9$. It is composed of 260 sensors with an internal voltage divider providing a drift field of $500 \mathrm{~V} / \mathrm{cm}$ and MOS charge injectors that allow measurement of the drift speed via dedicated calibration triggers.
The charge signal of each of the 133000 collection anodes, arranged with a pitch of $294 \mu \mathrm{m}$, is sampled every $50 \mathrm{~ns}$ by an ADC in the front-end electronics. The total thickness of the SDD layers (including mechanical supports and front-end electronics) amounts to $2.4 \%$ of a radiation length. About $92 \%$ of the anodes were fully operational.

- The two layers of the double-sided Silicon Strip Detector (SSD) are located at radii of 38 and $43 \mathrm{~cm}$ respectively, covering $|\eta|<0.97$. The SSD consists of 1698 sensors with a strip pitch of $95 \mu \mathrm{m}$ and a stereo angle of $35 \mathrm{mrad}$. The detector provides a measurement of the charge deposited in each of its $2.5 \times 10^{6}$ strips. The position resolution is better than $20 \mu \mathrm{m}$ in the $r-\varphi$ direction and about $0.8 \mathrm{~mm}$ in the direction along the beam line. The thickness of the SSD, including supports and services, corresponds to $2.2 \%$ of a radiation length. About $90 \%$ of the SSD area was active during data taking.

- The VZERO detector consists of two arrays of 32 scintillators each, which are placed around the beam pipe on either side of the interaction region: VZEROA at $z=3.3 \mathrm{~m}$, covering the pseudorapidity range $2.8<\eta<5.1$, VZERO-C at $z=-0.9 \mathrm{~m}$, covering the pseudorapidity range $-3.7<\eta<-1.7$. The time resolution of this detector is better than $1 \mathrm{~ns}$. Its response is recorded in a time window of $\pm 25 \mathrm{nsec}$ around the nominal beam crossing time. For events collected in this run, the arrival times of particles at the detector relative to this "time zero" is shown in Fig. 3. Note that in general several particles are registered for each event. Particles hitting one of the detectors before the beam crossing have negative arrival times and are typically due to interactions taking place outside the central region of ALICE.

More details about the ALICE experiment and its detector subsystems can be found in [2].

The trigger used to record the events for the present analysis is defined by requiring at least two hit chips in the SPD, in coincidence with the signals from the two beam pick-up counters indicating the presence of two passing proton bunches. The efficiency of this trigger as well as all other corrections have been studied using two different Monte Carlo generators, PYTHIA 6.4.14 [32, 33] tune D6T [34] and PHOJET [16], for INEL and NSD interactions. The trigger efficiencies for non-diffractive, singlediffractive, and double-diffractive events were evaluated separately, and found to be 98-99\%, 48-58\%, and 53-76\% respectively. The ranges are determined by the two event generators. These event classes were combined for the corrections using the fractions measured by UA5 [35]: nondiffractive $0.767 \pm 0.059$; single-diffractive $0.153 \pm 0.031$; double-diffractive $0.08 \pm 0.05$. The resulting efficiencies were found to be $87-91 \%$ for the INEL normalization and 


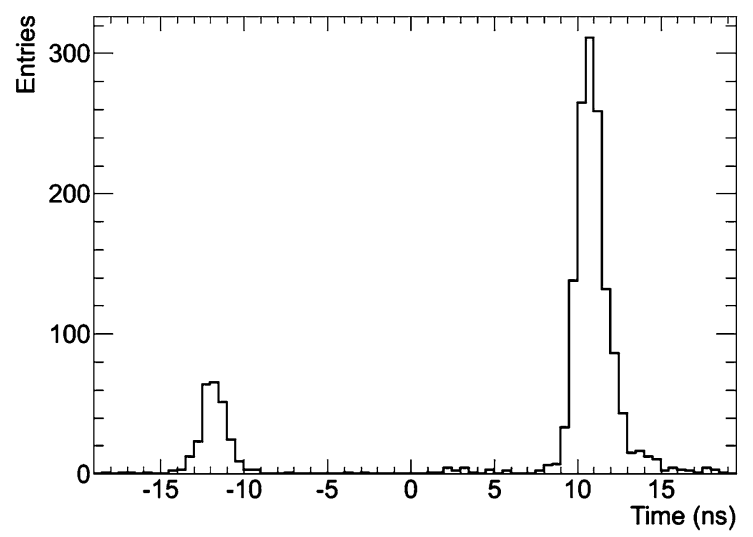

Fig. 3 Arrival time of particles in the VZERO detectors relative to the beam crossing time (time zero). A number of beam-halo or beam-gas events are visible as secondary peaks in VZERO-A (left panel) and VZERO-C (right panel). This is because particles produced in back-

94-97\% for the NSD normalization, again depending on the event generator used.

The results presented in the following sections are those obtained with PYTHIA. The difference between results corrected with PYTHIA and PHOJET is used in the estimate of the systematic uncertainty.

\section{Data analysis}

The data sample used in the present analysis consists of 284 events recorded without magnetic field. The results presented here are based on the analysis of the SPD data. However, information from the SDD, SSD and VZERO was used to crosscheck the identification and removal of background events.

In the SPD analysis, the position of the interaction vertex is reconstructed [36] by correlating hits in the two siliconpixel layers to obtain tracklets. The achieved resolution depends on the track multiplicity and for this specific vertex reconstruction is approximately $0.1-0.3 \mathrm{~mm}$ in the longitudinal direction and $0.2-0.5 \mathrm{~mm}$ in the transverse direction. For events with only one charged track, the vertex position is determined by intersecting the SPD tracklet with the mean beam axis determined from the vertex positions of other events in the sample. A vertex was reconstructed in $94 \%$ of the selected events. The distribution of the vertex position in the longitudinal direction ( $z$-axis) is shown in Fig. 4. For events originating from the centre of the detector, the vertex-reconstruction efficiency was estimated, using Monte Carlo simulations, to be $84 \%$ for INEL interactions and $92 \%$ for NSD collisions. These efficiencies decrease for larger $|z|$-values of the vertex in low-multiplicity events; therefore, only events with vertices within $|z|<10 \mathrm{~cm}$ were used. This allows for an accurate charged-particle density measurement in the pseudorapidity range $|\eta|<1.6$ using both SPD layers.

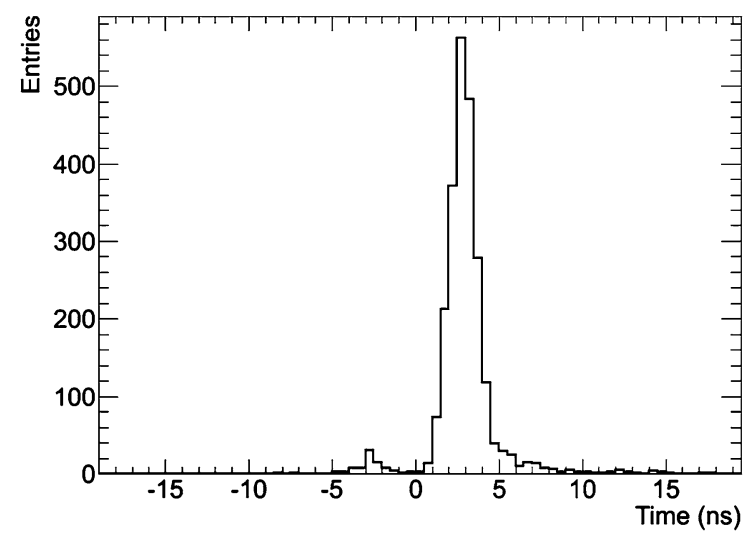

ground interactions arrive at earlier times in one or the other of the two counters. The majority of the signals have the correct arrival time expected for collisions around the nominal vertex

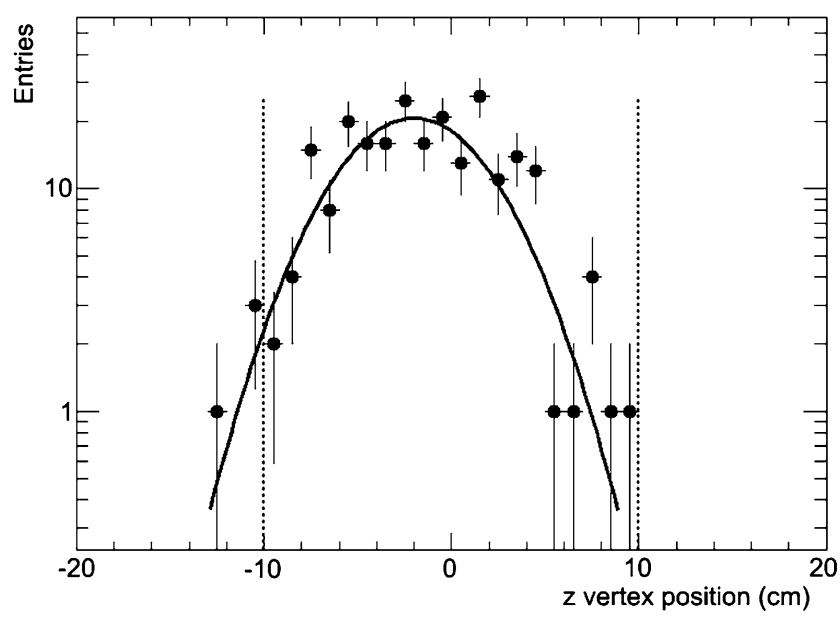

Fig. 4 Longitudinal vertex distribution from hit correlations in the two pixel layers of the ALICE inner tracking system. Vertical dashed lines indicate the region $|z|<10 \mathrm{~cm}$, where the events for the present analysis are selected. A Gaussian fit with an estimated r.m.s. of about $4 \mathrm{~cm}$ to the central part is also shown

Using the reconstructed vertex as the origin, we calculate the differences in azimuthal $(\Delta \varphi$, bending plane) and polar ( $\Delta \theta$, non-bending direction) angles of pairs of hits with one hit in each SPD layer. These tracklets [37] are selected by a cut on the sum of the squares of $\Delta \varphi$ and $\Delta \theta$, each normalized to its estimated resolution $(80 \mathrm{mrad}$ and $25 \mathrm{mrad}$, respectively). When more than one hit in a layer matches a hit in the other layer, only the hit combination with the smallest angular difference is used. This occurs in only $2 \%$ of the matched hits.

The number of primary charged particles is estimated by counting the number of tracklets. This number was corrected for:

- trigger inefficiency;

- detector and reconstruction inefficiencies; 
- contamination by decay products of long-lived particles $\left(\mathrm{K}_{\mathrm{s}}^{0}, \Lambda\right.$, etc. $)$, gamma conversions and secondary interactions.

The corrections are determined as a function of the $z$-position of the primary vertex, and on the pseudorapidity of the tracklet. For the analyzed sample the average correction factor for tracklets is about 1.5.

The beam-gas and beam-halo background events were removed by a cut on the ratio between the number of tracklets and the total number of hits in the tracking system (SPD, SDD, and SSD); this ratio is smaller for background events (as measured in the previous fills triggering on the bunch passage from one side) than for collisions [38]. In addition, the timing information from the VZERO detector was used for background rejection by removing events with negative arrival time (see Fig. 3). The event quality and event classification was crosschecked by a visual scan of the whole event sample. In total 29 events (i.e. about 10\%) were rejected as beam induced background, which is consistent with the rate expected from previous fills. The remaining background was estimated from the vertex distribution and found to be negligible. The contamination from coincidence with a cosmic event was estimated to be one event in the full sample. Indeed, two cosmic events were identified by scanning, both without reconstructed vertex.

Particular attention has been paid to events having zero or one charged tracklets in the SPD acceptance. The vertexfinding efficiency for events with one charged particle in the acceptance is about $80 \%$. The number of zero-track events has been estimated by Monte Carlo calculations. The total number of collisions used for the normalization was calculated from the number of events selected for the analysis, corrected for the vertex-reconstruction inefficiency. In order to obtain the normalization for INEL and NSD events, we further corrected the number of selected events for the trigger efficiency for these two event classes. In addition, for NSD events, we subtract the single-diffractive contribution. These corrections, as well as those for the vertex finding efficiency, depend on the event charged-particle multiplicity, see Fig. 5. The dependence of the event-finding efficiency (combining event selection and vertex finding) on multiplicity was calculated for different interaction types using our detector simulation, and is above $98 \%$ for events with at least two charged particles. The averaged combined corrections for the vertex reconstruction efficiency and the selection efficiency is $20 \%$ for INEL interactions and much smaller for NSD interactions, due to the cancelation of some contributions.

The various corrections mentioned above were calculated using the full GEANT $3[39,40]$ simulation of the ALICE detector as included in the offline framework AliRoot. In order to estimate the systematic uncertainties, the above analysis was repeated by:

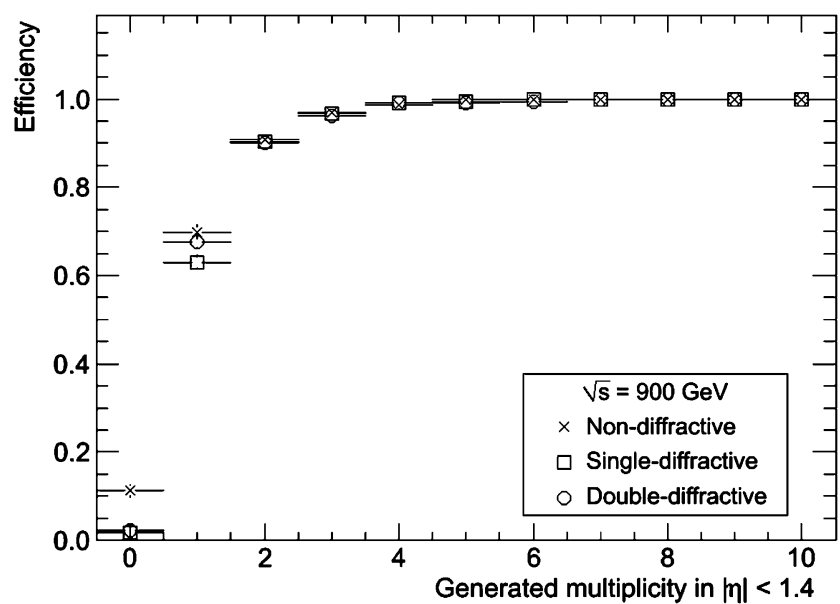

Fig. 5 Multiplicity dependence of the combined efficiency to select an event as minimum bias and to reconstruct its vertex in SPD, for non-diffractive (crosses), single-diffractive (squares), and double-diffractive (circles) events, based on PYTHIA events

- applying different cuts for the tracklet definition (varying the angle cut-off by $\pm 50 \%$ );

- varying by $\pm 10 \%$ the density of the material in the tracking system, thus changing the material budget;

- using the non-aligned geometry;

- varying by $\pm 30 \%$ the composition of the produced particle types with respect to the yields suggested by the event generators;

- varying the particle yield below $100 \mathrm{MeV} / \mathrm{c}$ by $\pm 30 \%$;

- evaluating the uncertainty in the normalization to INEL and NSD samples by varying the ratios of the non-diffractive, single-diffractive and double-diffractive cross sections according to their measured values and errors [35] and using two different models for diffraction kinematics (PYTHIA and PHOJET).

An additional source of systematic error comes from the limited statistics used so far to determine the efficiencies of the SPD detector modules. In test beams, the SPD efficiency in active areas was measured to be higher than $99.8 \%$. This was crosschecked in-situ with cosmic data, but only over a limited area and with limited statistics. At this stage, we have assigned a conservative value of $4 \%$ to this uncertainty. The triggering efficiency of the SPD was estimated from the data itself, using the trigger information recorded in the data stream for events with more than one tracklet, and found to be very close to $100 \%$, with an error of about $2 \%$ (due to the limited statistics).

These contributions to the systematic uncertainty on the charged particle pseudorapidity density are summarized in Table 1. Our conclusion is that the total systematic uncertainty on the pseudorapidity density is less than $\pm 7.2 \%$ for INEL collisions and $\pm 7.1 \%$ for NSD collisions. The largest contribution comes from uncertainties in cross sections of diffractive processes and their kinematic simulation. 
Table 1 Contributions to systematic uncertainties on the measurement of the charged-particle pseudorapidity density

\begin{tabular}{ll}
\hline Uncertainty & \\
\hline Tracklet selection cuts & negl. \\
Material budget & negl. \\
Misalignment & $0.5 \%$ \\
Particle composition & negl. \\
Transverse-momentum spectrum & $0.5 \%$ \\
Contribution of diffraction (INEL) & $4 \%$ \\
Contribution of diffraction (NSD) & $4.5 \%$ \\
Event-generator dependence (INEL) & $4 \%$ \\
Event-generator dependence (NSD) & $3 \%$ \\
Detector efficiency & $4 \%$ \\
SPD triggering efficiency & $2 \%$ \\
Background events & negl. \\
\hline Total (INEL) & $7.2 \%$ \\
Total (NSD) & $7.1 \%$ \\
\hline
\end{tabular}

More details about this analysis, corrections, and the evaluation of the systematic uncertainties can be found in [41].

\section{Results}

Figure 6 shows the charged primary particle pseudorapidity density distributions obtained for INEL and NSD interactions in the range $|\eta|<1.6$. The pseudorapidity density obtained in the central region $|\eta|<0.5$ for INEL interactions is $3.10 \pm 0.13$ (stat.) \pm 0.22 (syst.) and for NSD interactions is $3.51 \pm 0.15$ (stat.) \pm 0.25 (syst.). Also shown in Fig. 6 are the previous measurements of proton-antiproton interactions from the UA5 experiment [3]. Our results obtained for proton-proton interactions are consistent with those for proton-antiproton interactions, as expected from the fact that the predicted difference $(0.1-0.2 \%)$ is well below measurement uncertainties. The measurements at central pseudorapidity $(|\eta|<0.5)$ are summarized in Table 2 together with model predictions obtained with QGSM, PHO-

Table 2 Comparison of charged primary particle pseudorapidity densities at central pseudorapidity $(|\eta|<0.5)$ for inelastic (INEL) and non-single diffractive (NSD) collisions measured be the ALICE detector in pp interactions and by UA5 in $\mathrm{p} \overline{\mathrm{p}}$ interactions [3] at a centreof-mass energy of $900 \mathrm{GeV}$. For ALICE, the first error is statistical

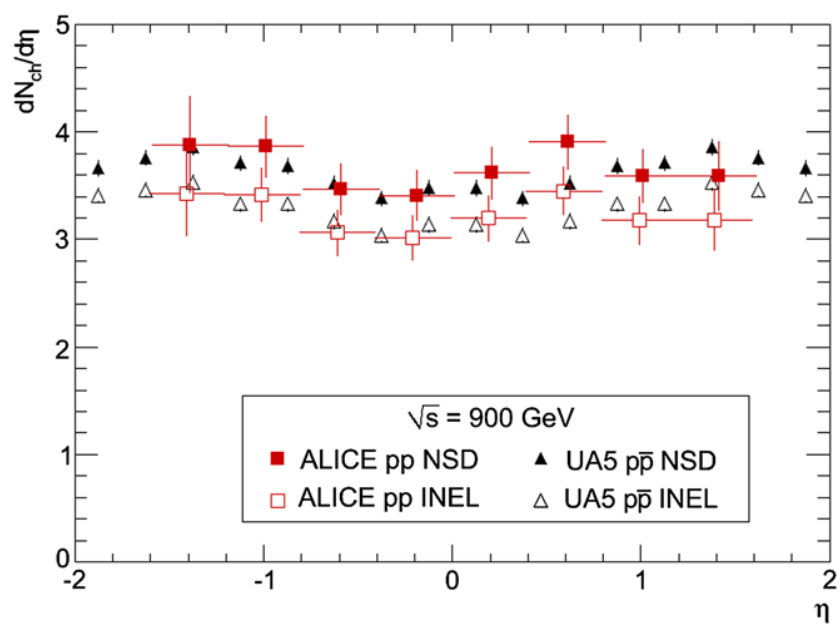

Fig. 6 Pseudorapidity dependence of $\mathrm{d} N_{\mathrm{ch}} / \mathrm{d} \eta$ for INEL and NSD collisions. The ALICE measurements (squares) are compared to UA5 data (triangles) [3]. The errors shown are statistical only

JET and three different PYTHIA tunes. PYTHIA 6.4.14, tune D6T, and PHOJET yield respectively the lowest and highest charged particle densities. Therefore, these two have been used for the evaluation of our systematic errors. PYTHIA 6.4.20, tunes ATLAS CSC and Perugia-0, are candidates for use by the LHC experiments at higher LHC energies and are shown for comparison.

Figure 7 shows the centre-of-mass energy dependence of the pseudorapidity density in the central region $(|\eta|<0.5)$. The data points are obtained in the $|\eta|<0.5$ range from this experiment and from references [3, 18, 19, 45-48], and are corrected for differences in pseudorapidity range where necessary, fitting the pseudorapidity distribution around $\eta=0$. As noted above, there is good agreement between pp and $\mathrm{p} \overline{\mathrm{p}}$ data at the same energy. The dashed and solid lines (for INEL and NSD interactions respectively) are obtained by fitting the density of charged particles in the central pseudorapidity rapidity region with a power-law dependence on energy.

Using this parametrization, the extrapolation to the nominal LHC energy of $\sqrt{s}=14 \mathrm{TeV}$ yields $\mathrm{d} N_{\mathrm{ch}} / \mathrm{d} \eta=5.5$ and $\mathrm{d} N_{\mathrm{ch}} / \mathrm{d} \eta=5.9$ for INEL and NSD interactions respectively.

and the second is systematic; no systematic error is quoted by UA5. The experimental data are also compared to the predictions for pp collisions from different models. For PYTHIA the tune versions are given in parentheses. The correspondence is as follows: D6T is tune (109); ATLAS CSC is tune (306); Perugia-0 is tune (320)

\begin{tabular}{|c|c|c|c|c|c|c|c|}
\hline \multirow{2}{*}{$\begin{array}{l}\text { Experiment } \\
\text { Model }\end{array}$} & \multirow[t]{2}{*}{ ALICE pp } & \multirow[t]{2}{*}{ UA5 p p $[3]$} & \multirow[t]{2}{*}{ QGSM [42] } & \multicolumn{3}{|c|}{ PYTHIA $[32,33]$} & \multirow[t]{2}{*}{ PHOJET [16] } \\
\hline & & & & (109) [34] & (306) [43] & (320) [44] & \\
\hline INEL & $3.10 \pm 0.13 \pm 0.22$ & $3.09 \pm 0.05$ & 2.98 & 2.33 & 2.99 & 2.46 & 3.14 \\
\hline NSD & $3.51 \pm 0.15 \pm 0.25$ & $3.43 \pm 0.05$ & 3.47 & 2.83 & 3.68 & 3.02 & 3.61 \\
\hline
\end{tabular}




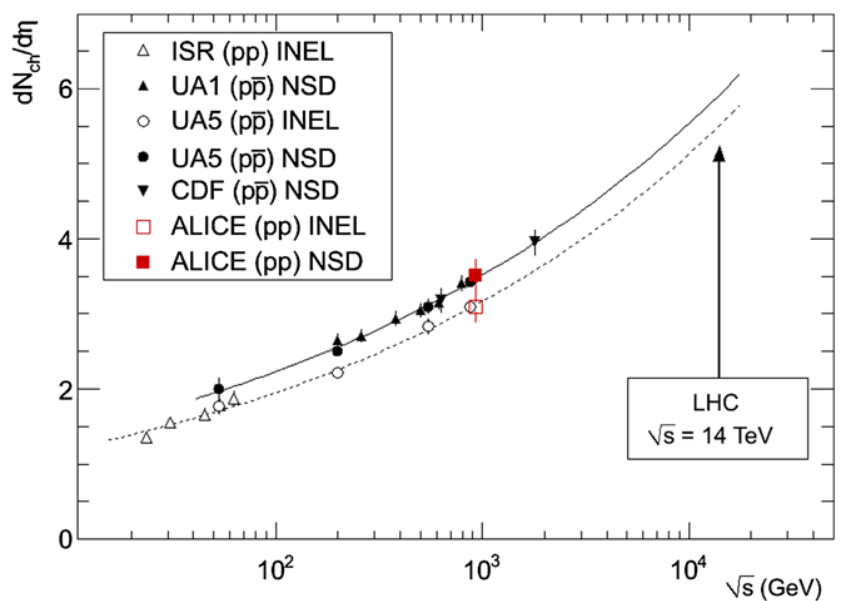

Fig. 7 Charged-particle pseudorapidity density in the central rapidity region in proton-proton and proton-antiproton interactions as a function of the centre-of-mass energy. The UA5 and ALICE data points at $900 \mathrm{GeV}$ are slightly displaced horizontally for clarity. The dashed and solid lines (for INEL and NSD interactions respectively) indicate the fit using a power-law dependence on energy

\section{Conclusion}

Proton-proton collisions observed with the ALICE detector in the early phase of the LHC commissioning have been used to measure the pseudorapidity density of charged primary particles at $\sqrt{s}=900 \mathrm{GeV}$. In the central pseudorapidity region $(|\eta|<0.5)$, we obtain $\mathrm{d} N_{\mathrm{ch}} / \mathrm{d} \eta=3.10 \pm$ 0.13 (stat.) \pm 0.22 (syst.) for all inelastic and $\mathrm{d} N_{\mathrm{ch}} / \mathrm{d} \eta=$ $3.51 \pm 0.15$ (stat.) \pm 0.25 (syst.) for non-single diffractive proton-proton interactions. The results are consistent with earlier measurements of primary charged-particle production in proton-antiproton interactions at the same energy. They are also compared with model calculations.

These results have been obtained with a small sample of events during the early commissioning of the LHC. They demonstrate that the LHC and its experiments have finally entered the phase of physics exploitation, within days of starting up the accelerator complex in November 2009.

Acknowledgement The ALICE collaboration would like to thank all its engineers and technicians for their invaluable contributions to the construction of the experiment. We would like to thank and congratulate the CERN accelerator teams for the outstanding performance of the LHC complex at start up, and for providing us with the collisions used for this paper on such a short notice!

The ALICE collaboration acknowledges the following funding agencies for their support in building and running the ALICE detector:

- Calouste Gulbenkian Foundation from Lisbon and Swiss Fonds Kidagan, Armenia;

- Conselho Nacional de Desenvolvimento Científico e Tecnológico (CNPq), Financiadora de Estudos e Projeto (FINEP), Fundação de Amparo à Pesquisa do Estado de São Paulo (FAPESP);

- National Natural Science Foundation of China (NSFC), the Chinese Ministry of Education (CMOE) and the Ministry of Science and Technology of China (MSTC);
- Ministry of Education and Youth of the Czech Republic;

- Danish National Science Research Council and the Carlsberg Foundation;

- The European Research Council under the European Community's Seventh Framework Programme;

- Helsinki Institute of Physics and the Academy of Finland;

- French CNRS-IN2P3, the 'Region Pays de Loire', 'Region Alsace', 'Region Auvergne' and CEA, France;

- German BMBF and the Helmholtz Association;

- Hungarian OTKA and National Office for Research and Technology (NKTH);

- Department of Atomic Energy and Department of Science and Technology of the Government of India;

- Istituto Nazionale di Fisica Nucleare (INFN) of Italy;

- MEXT Grant-in-Aid for Specially Promoted Research, Japan;

- Joint Institute for Nuclear Research, Dubna;

- Korea Foundation for International Cooperation of Science and Technology (KICOS);

- CONACYT, DGAPA, México, ALFA-EC and the HELEN Program (High-Energy physics Latin-American-European Network);

- Stichting voor Fundamenteel Onderzoek der Materie (FOM) and the Nederlandse Organistie voor Wetenschappelijk Onderzoek (NWO), Netherlands;

- Research Council of Norway (NFR);

- Polish Ministry of Science and Higher Education;

- National Authority for Scientific Research-NASR (Autontatea Nationala pentru Cercetare Stiintifica-ANCS);

- Federal Agency of Science of the Ministry of Education and Science of Russian Federation, International Science and Technology Center, Russian Federal Agency of Atomic Energy, Russian Federal Agency for Science and Innovations and CERN-INTAS;

- Ministry of Education of Slovakia;

- CIEMAT, EELA, Ministerio de Educación y Ciencia of Spain, Xunta de Galicia (Consellería de Educación), CEADEN, Cubaenergía, Cuba, and IAEA (International Atomic Energy Agency);

- Swedish Research Council (VR) and Knut \& Alice Wallenberg Foundation (KAW);

- Ukraine Ministry of Education and Science;

- United Kingdom Science and Technology Facilities Council (STFC);

- The United States Department of Energy, the United States National Science Foundation, the State of Texas, and the State of Ohio.

Open Access This article is distributed under the terms of the Creative Commons Attribution Noncommercial License which permits any noncommercial use, distribution, and reproduction in any medium, provided the original author(s) and source are credited.

\section{References}

1. L. Evans, P. Bryant, J. Instrum. 3, S08001 (2008)

2. K. Aamodt et al. (ALICE Collaboration), J. Instrum. 3, S08002 (2008)

3. G.J. Alner et al. (UA5 Collaboration), Z. Phys. C 33, 1 (1986)

4. A.B. Kaidalov, Phys. Lett. B 116, 459 (1982)

5. A.B. Kaidalov, K.A. Ter-Martirosyan, Phys. Lett. B 117, 247 (1982)

6. A.B. Kaidalov, K.A. Ter-Martirosyan, Yad. Fiz. 39, 1545 (1984) (Sov. J. Nucl. Phys. 39, 979 (1984))

7. A.B. Kaidalov, K.A. Ter-Martirosyan, Yad. Fiz. 40, 211 (1984) (Sov. J. Nucl. Phys. 40, 135 (1984))

8. A. Capella et al., Z. Phys. C 3, 329 (1980)

9. A. Capella, J. Tran Thanh Van, Z. Phys. C 10, 249 (1981)

10. A. Capella, J. Tran Thanh Van, Phys. Lett. B 114, 450 (1982) 
11. A. Capella, U. Sukhatme, C.-I. Tan, J. Tran Thanh Van, Phys. Rep. 236, 225 (1994)

12. N.S. Amelin, L.V. Bravina, Yad. Fiz. 51, 211 (1990) (Sov. J. Nucl. Phys. 51, 133 (1990))

13. N.S. Amelin et al., Yad. Fiz. 51, 512 (1990) (Sov. J. Nucl. Phys. 51, 327 (1990))

14. N.S. Amelin et al., Yad. Fiz. 52, 272 (1990) (Sov. J. Nucl. Phys. 51, 172 (1990))

15. P. Aurenche et al., Phys. Rev. D 45, 92 (1992)

16. R. Engel, J. Ranft, S. Roesler, Phys. Rev. D 52, 1459 (1995)

17. J.G. Rushbrooke, B.R. Webber, Phys. Rep. C 44, 1 (1978)

18. K. Alpgård et al. (UA5 Collaboration), Phys. Lett. B 112, 183 (1982)

19. M. Ambrosio et al., AIP Conf. Proc. 85, 602 (1982)

20. T. Antičić et al., ALICE Internal Note ALICE-INT-2005-015 (2005)

21. T. Antičić et al. (ALICE Collaboration), J. Phys. Conf. Ser. 119, 022006 (2008)

22. (ALICE Collaboration) AliRoot, ALICE Offline simulation, reconstruction and analysis framework, http://aliceinfo.cern. ch/Offline/

23. T.M. Steinbeck (ALICE Collaboration), in Proceedings of CHEP 2009, March 2009, Prague. J. Phys. Conf. Ser., to appear

24. ALICE Collaboration, J. Phys. G 30, 1517 (2004)

25. ALICE Collaboration J. Phys. G 32, 1295 (2006)

26. P.G. Kuijer (ALICE Collaboration), Nucl. Phys. A 830, 81C (2009)

27. F. Prino (ALICE Collaboration), Nucl. Phys. A 830, 527C (2009)

28. R. Santoro et al. (ALICE Collaboration), J. Instrum. 4, P03023 (2009)

29. G. Aglieri Rinella et al., CERN preprint CERN-2008-008, 2008

30. P. Giubellino (ALICE Collaboration), in Proceedings of EPS HEP 2009, July 2009, Krakow. Proc. Sci., to appear
31. C. Bombonati et al., ALICE Internal Note ALICE-INT-2009-035, 2009

32. T. Sjöstrand, Comput. Phys. Commun. 82, 74 (1994)

33. T. Sjöstrand, S. Mrenna, P. Skands, J. High Energy Phys. 2006, 05 (2006). 026

34. M.G. Albrow et al. (Tev4LHC QCD Working Group), arXiv:hep-ph/0610012 (2006), D6T (109) tune

35. R.E. Ansorge et al. (UA5 Collaboration), Z. Phys. C 33, 175 (1986)

36. E. Bruna et al., ALICE Internal Note ALICE-INT-2009-018, 2009

37. D. Elia et al., ALICE Internal Note ALICE-INT-2009-021, 2009

38. J.F. Grosse-Oetringhaus et al., ALICE Internal Note ALICE-INT2009-022, 2009

39. R. Brun et al., GEANT3 User Guide CERN, Data Handling Division DD/EE/841, 1985

40. R. Brun et al., CERN Program Library Long Write-up, W5013, GEANT Detector Description and Simulation Tool, 1994

41. J.F. Grosse-Oetringhaus, Ph.D. thesis, University of Münster, Germany, 2009. CERN-THESIS-2009-033

42. A.B. Kaidalov, M.G. Poghosyan, Eur. Phys. J. C, submitted. arXiv:0910.2050 [hep-ph] (2009)

43. A. Moraes (ATLAS Collaboration), ATLAS Note ATL-COMPHYS-2009-119, 2009. ATLAS CSC (306) tune

44. P.Z. Skands, in Multi-Parton Interaction Workshop, Perugia, Italy, 28-31 Oct. 2008. arXiv:0905.3418 [hep-ph] (2009), Perugia-0 (320) tune

45. W. Thome et al., Nucl. Phys. B 129, 365 (1977)

46. C. Albajar et al. (UA1 Collaboration), Nucl. Phys. B 335, 261 (1990)

47. G.J. Alner et al. (UA5 Collaboration), Phys. Rep. 154, 247 (1987)

48. F. Abe et al. (CDF Collaboration), Phys. Rev. D 41, 2330 (1990) 